\title{
PERMANENT CONFIGURATIONS IN THE PROBLEM OF FOUR BODIES*
}

\author{
BY
}

W. D. MACMILLAN AND WALTER BARTKY

1. Introduction. Two permanent configurations in the problem of three bodies have been known since the time of Lagrange, namely, the straight line and the equilateral triangle; each of these configurations exists whatever the masses may be. A complete generalization of the straight line configuration to $n$ bodies was given by F. R. Moulton, $\dagger$ and some particular instances of other configurations have been given by $\mathrm{R}$. Hoppe, $\ddagger$ Andoyer, $\$$ and W. R. Longley, \| each of which contains some element of symmetry.

In the present paper the problem of plane configurations (evidently, with the exception of the tetrahedron, there are no three-dimensional configurations) is removed from the field of differential equations to those of geometry and algebra by means of two theorems which hold for any number of bodies. The results of these theorems are used to give a complete and detailed analysis of the quadrilateral configurations of four bodies. It is evident that the method is applicable to any number of bodies.

2. The differential equations. Suppose there are $n$ particles in the $x y$-plane which attract each other along the lines joining them according to any given function of the distance, but which, for simplicity, will be taken to be the inverse $n$th power of the distance. The differential equations, referred to the center of gravity of the system, are

$$
\begin{aligned}
& x_{i}^{\prime \prime}=-\sum m_{j} \frac{x_{i}-x_{j}}{r_{i j}{ }^{n+1}}=-\sum m_{j} \frac{\cos \theta_{i j}}{r_{i j}{ }^{n}}, \\
& y_{i}^{\prime \prime}=-\sum m_{j} \frac{y_{i}-y_{j}}{r_{i j}{ }^{n+1}}=-\sum m_{j} \frac{\sin \theta_{i j}}{r_{i j}{ }^{n}}(i=1, \cdots, n ; j \neq i) ;
\end{aligned}
$$

or, in polar coördinates, in which

$$
x_{i}=r_{i} \cos \theta_{i}, \quad y_{i}=r_{i} \sin \theta_{i j}
$$

* Presented to the Society, September 2, 1932; received by the editors May 16, 1932.

$\dagger$ Periodic Orbits, published by the Carnegie Institution of Washington, 1920, p. 285.

$\ddagger$ Erweiterung der bekannten Speciallösung des Dreikörper problems, Archiv der Mathematik und Physik, vol. 64, p. 218.

$\S$ Sur l'équilibre relatif de $n$ corps, Bulletin Astronomique, vol. 23 (1906), p. 50.

II Some particular solutions in the problem of $n$ bodies, Bulletin of the American Mathematical Society, vol. 13 (1906-07), p. 324. 
the equations are

$$
\begin{aligned}
r_{i}^{\prime \prime}-r_{i} \theta_{i}^{\prime 2} & =-\sum m_{j} \frac{\cos \left(\theta_{i j}-\theta_{i}\right)}{r_{i j}{ }^{n}}, \\
r_{i} \theta_{i}^{\prime \prime}+2 r_{i}^{\prime} \theta_{i}^{\prime} & =-\sum m_{j} \frac{\sin \left(\theta_{i j}-\theta_{i}\right)}{r_{i j}{ }^{n}} .
\end{aligned}
$$

In these equations $\theta_{i j}$ is the angle which the line $r_{i j}$ makes with the $x$-axis.

If there exists a configuration which moves like a rigid system with the angular velocity $\omega$, the mutual distances are all constants, say

$$
r_{i}=l_{i}, \quad r_{i j}=l_{i j}
$$

and the angles are all linear functions of the time, say

$$
\theta_{i}=\theta_{i}{ }^{(0)}+\omega t, \quad \theta_{i j}=\theta_{i j}{ }^{(0)}+\omega t .
$$

The differential equations reduce to

$$
\begin{aligned}
& \sum m_{j} \frac{\cos \left(\theta_{i j}{ }^{(0)}-\theta_{i}{ }^{(0)}\right)}{l_{i j}{ }^{n}}=\omega^{2} l_{i}, \\
& \sum m_{j} \frac{\sin \left(\theta_{i j}{ }^{(0)}-\theta_{i}^{(0)}\right)}{l_{i j}{ }^{n}}=0 \quad(i=1, \cdots, n) .
\end{aligned}
$$

The left members of these equations are the components of acceleration of the particle $m_{i}$, along the radius vector $r_{i}$ and perpendicular to it, due to the attraction of all of the other bodies. Expressed in words these equations give the following theorem:

THEOREM I. If a plane system of free particles, which is acted upon by no forces other than those of their mutual attractions, rotates about the center of gravity like a rigid system then the resultant acceleration of each of the particles, due to the attraction of all of the other particles, passes through the center of gravity of the system, and in magnitude is proportional to the distance of the particle from the center of gravity of the system; and, conversely, if there exists a plane configuration of $n$ bodies in which the resultant acceleration of each particle passes through the center of gravity of the system and in magnitude is proportional to the distance of the particle from the center of gravity, then the system in this configuration can rotate like a rigid system.

3. The possibility of Keplerian motion. The hypothesis of rigidity is stronger than is necessary, for the configuration is preserved if the system is altered in such a way that the ratios of the mutual distances are not changed; size and orientation being non-essentials. 
Suppose one knows a configuration in which the resultant acceleration of each particle, due to the attraction of all of the others, is directed toward the center of gravity and in magnitude is proportional to the distance of the particle from the center of gravity, and equations (2), therefore, are satisfied.

Let $\rho$ and $\theta$ be new variables, and in equations (1) take

$$
\begin{aligned}
r_{i} & =\rho l_{i}, & r_{i j} & =\rho l_{i j}, \\
\theta_{i} & =\theta_{i}{ }^{(0)}+\theta, & \theta_{i j} & =\theta_{i j}{ }^{(0)}+\theta .
\end{aligned}
$$

Equations (1) then become

$$
\begin{gathered}
l_{i} \rho^{\prime \prime}-l_{i} \rho \theta^{\prime 2}=-\frac{1}{\rho^{n}} \sum m_{j} \frac{\cos \left(\theta_{i j}{ }^{(0)}-\theta_{i}{ }^{(0)}\right)}{l_{i j}{ }^{n}}, \\
l_{i}\left(\rho \theta^{\prime \prime}+2 \rho^{\prime} \theta^{\prime}\right)=0,
\end{gathered}
$$

which, by virtue of equations (2) and removal of the factor $l_{i}$, become

$$
\begin{aligned}
\rho^{\prime \prime}-\rho \theta^{\prime 2} & =-\frac{\omega^{2}}{\rho^{n}}, \\
\rho \theta^{\prime \prime}+2 \rho^{\prime} \theta^{\prime} & =0 .
\end{aligned}
$$

These are the equations of motion of a particle which is attracted towards a fixed center by a force which varies inversely as the $n$th power of the distance. Hence

THEOREM II. If a configuration of $n$ particles exists for which motion as a rigid system is possible, then each particle of the system can move just as though it were attracted toward the center of gravity by a force which varies inversely as the nth power of the distance in such a way that the configuration is preserved.

If the law of attraction is the Newtonian law and the configuration is such that circular motion is possible, then motion in a conic section in accordance with the laws of Kepler also is possible, the configuration being preserved throughout the motion.

\section{PERMANENT QUADRILATERAL CONFIGURATIONS}

4. Vector equations. Given four masses, $m_{1}, m_{2}, m_{3}, m_{4}$, and the quadrilateral of which they are the corners, Fig. 1 . Let the line $m_{1} m_{2}$ be $r_{1}, m_{2} m_{3}$ be $r_{2}$, etc.; let the diagonal $m_{1} m_{3}$ be $r_{5}$ and the diagonal $m_{2} m_{4}$ be $r_{6}$. Let $\mathbf{a}_{1}, \boldsymbol{a}_{2}, \mathbf{a}_{3}, \mathbf{a}_{4}$ be unit vectors parallel to $r_{1}, r_{2}, r_{3}$, and $r_{4}$ respectively, taken as in the figure. The sides $r_{1}$ and $r_{3}$ intersect in the point $A$, and the sides $r_{2}$ and $r_{4}$ in the point $B$. Starting at $m_{i}$, let $\alpha_{i} r_{i}$ be the distance along the line $r_{i}$ to the intersection with the opposite side (the point $A$ or $B$ ). The ratio $\alpha_{i}$ is positive if this direction is the same as that of $a_{i}$; otherwise, negative; and let $\beta_{i}=\alpha_{i}-1$. 
If $L_{i+1}$ is the line which coincides with the side $r_{i+1}$ of the quadrilateral, and if $I_{i}$ is a variable vector with its origin at $m_{i}$, and $t_{i}$ is a variable parameter, the vector equations of the lines $L_{i+1}$ are

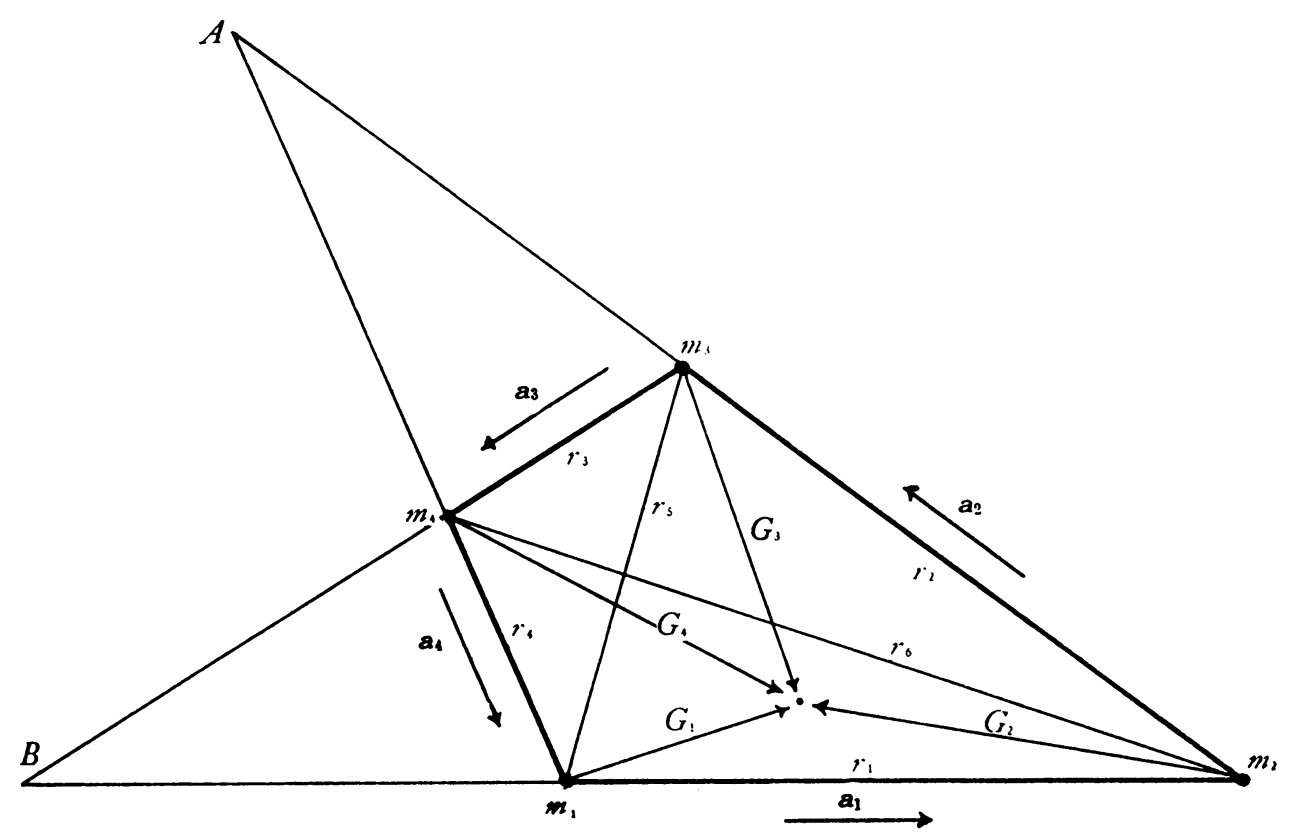

Fig. 1

(3)

$$
\begin{aligned}
& I_{1}=t_{1} r_{1} a_{1}+\left(1-t_{1}\right) \beta_{4} r_{4} a_{4}, \\
& I_{2}=t_{2} r_{2} a_{2}+\left(1-t_{2}\right) \beta_{1} r_{1} a_{1}, \\
& I_{3}=t_{3} r_{3} a_{3}+\left(1-t_{3}\right) \beta_{2} r_{2} a_{2}, \\
& I_{4}=t_{4} r_{4} a_{4}+\left(1-t_{4}\right) \beta_{3} r_{3} a_{3} \quad\left(\beta_{i}=\alpha_{i}-1, \alpha_{i}-\beta_{i}=+1\right) .
\end{aligned}
$$

For certain values of the parameters $t_{i}$ the vectors $I_{i}$ coincide with the diagonals of the quadrilateral. These values are

$$
t_{i}=\frac{\beta_{i+1}}{\alpha_{i+1}}, \quad 1-t_{i}=\frac{1}{\alpha_{i+1}}, \text { at the point } m_{i+2} ;
$$

and therefore the vector expressions for the diagonals are

$$
\begin{aligned}
I_{13} & =\frac{1}{\alpha_{2}}\left[\beta_{2} r_{1} a_{1}+\beta_{4} r_{4} a_{4}\right], & I_{31} & =\frac{1}{\alpha_{4}}\left[\beta_{4} r_{3} a_{3}+\beta_{2} r_{2} a_{2}\right], \\
I_{24} & =\frac{1}{\alpha_{3}}\left[\beta_{3} r_{2} a_{2}+\beta_{1} r_{1} a_{1}\right], & I_{42} & =\frac{1}{\alpha_{1}}\left[\beta_{1} r_{4} a_{4}+\beta_{3} r_{3} a_{3}\right] .
\end{aligned}
$$


Of course,

$$
I_{13}+I_{31}=0, \quad I_{24}+I_{42}=0 .
$$

Let $M$ be the sum of the four masses, and let $G_{1}, G_{2}, G_{3}$, and $G_{4}$ be the vectors which represent the center of gravity of the system with respect to the masses $m_{1}, m_{2}, m_{3}$, and $m_{4}$ respectively. Then

$$
\begin{aligned}
& M G_{1}=m_{2} r_{1} \mathbf{a}_{1}+m_{3} I_{13}-m_{4} r_{4} a_{4}, \\
& M G_{2}=m_{3} r_{2} a_{2}+m_{4} I_{24}-m_{1} r_{1} a_{1}, \\
& M G_{3}=m_{4} r_{3} a_{3}+m_{1} I_{31}-m_{2} r_{2} \mathbf{a}_{2}, \\
& M G_{4}=m_{1} r_{4} \boldsymbol{a}_{4}+m_{2} I_{42}-m_{3} r_{3} a_{3} ;
\end{aligned}
$$

and the substitution of (4) in (5) gives

(6)

$$
\begin{aligned}
& M G_{1}=\frac{1}{\alpha_{2}}\left[\left(\alpha_{2} m_{2}+\beta_{2} m_{3}\right) r_{1} a_{1}+\left(\beta_{4} m_{3}-\alpha_{2} m_{4}\right) r_{4} a_{4}\right], \\
& M G_{2}=\frac{1}{\alpha_{3}}\left[\left(\alpha_{3} m_{3}+\beta_{3} m_{4}\right) r_{2} a_{2}+\left(\beta_{1} m_{4}-\alpha_{3} m_{1}\right) r_{1} a_{1}\right], \\
& M G_{3}=\frac{1}{\alpha_{4}}\left[\left(\alpha_{4} m_{4}+\beta_{4} m_{1}\right) r_{3} a_{3}+\left(\beta_{2} m_{1}-\alpha_{4} m_{2}\right) r_{2} a_{2}\right], \\
& M G_{4}=\frac{1}{\alpha_{1}}\left[\left(\alpha_{1} m_{1}+\beta_{1} m_{2}\right) r_{4} a_{4}+\left(\beta_{3} m_{2}-\alpha_{1} m_{3}\right) r_{3} a_{3}\right] .
\end{aligned}
$$

5. Relations among the $\alpha$ 's. From the triangle $m_{1} m_{2} B$ it is found that

and similarly

$$
r_{2} a_{2}=-\frac{1}{\alpha_{2}} r_{1} a_{1}+\frac{\beta_{4}}{\alpha_{2}} r_{4} a_{4}
$$

$$
r_{3} a_{3}=-\frac{1}{\alpha_{3}} r_{2} a_{2}+\frac{\beta_{1}}{\alpha_{3}} r_{1} a_{1} .
$$

These values substituted in the equation

give the equation

$$
r_{1} a_{1}+r_{2} a_{2}+r_{3} a_{3}+r_{4} a_{4}=0
$$

$$
\frac{1}{\alpha_{2} \alpha_{3}}\left[\left(\beta_{2} \beta_{3}+\alpha_{1} \alpha_{2}\right) r_{1} a_{1}+\left(\beta_{3} \beta_{4}+\alpha_{2} \alpha_{3}\right) r_{4} a_{4}\right]=0 .
$$

Since $a_{1}$ and $a_{4}$ are non-collinear vectors, it follows that, if $\alpha_{2} \alpha_{3} \neq \infty$,

$$
\begin{aligned}
& \alpha_{1} \alpha_{2}+\beta_{2} \beta_{3}=0, \\
& \alpha_{2} \alpha_{3}+\beta_{3} \beta_{4}=0 ;
\end{aligned}
$$


and, similarly,

$$
\begin{aligned}
& \alpha_{3} \alpha_{4}+\beta_{4} \beta_{1}=0, \\
& \alpha_{4} \alpha_{1}+\beta_{1} \beta_{2}=0 .
\end{aligned}
$$

Only two of these relations are independent, for if the first and fourth are solved for $\alpha_{3}$ and $\alpha_{4}$ and the results substituted in the second and third, both equations are satisfied identically.

From the differences of these equations one derives also

$$
\begin{array}{ll}
\beta_{1} \alpha_{2}+\beta_{2} \alpha_{3}=-1, \\
\alpha_{2} \beta_{1}=\alpha_{4} \beta_{3}, \quad \beta_{2} \alpha_{3}+\beta_{3} \alpha_{4}=-1, \\
\alpha_{3} \beta_{2}=\alpha_{1} \beta_{4}, \quad \beta_{3} \alpha_{4}+\beta_{4} \alpha_{1}=-1, \\
& \beta_{4} \alpha_{1}+\beta_{1} \alpha_{2}=-1 .
\end{array}
$$

6. Relations between sides and diagonals. From the triangles $m_{1} m_{2} m_{3}$, $B m_{1} m_{3}$, and $A m_{2} m_{3}$ are obtained

$$
\begin{aligned}
r_{5}^{2} & =r_{1}^{2}+r_{2}^{2}-2 r_{1} r_{2} \cos \left(r_{1} r_{2}\right), \\
\alpha_{3}^{2} r_{3}^{2} & =\beta_{1}^{2} r_{1}^{2}+r_{2}^{2}+2 \beta_{1} r_{1} r_{2} \cos \left(r_{1} r_{2}\right), \\
\beta_{4}^{2} r_{4}^{2} & =r_{1}^{2}+\alpha_{2}^{2} r_{2}^{2}-2 \alpha_{2} r_{1} r_{2} \cos \left(r_{1} r_{2}\right) .
\end{aligned}
$$

The elimination of $\cos \left(r_{1} r_{2}\right)$ between the first and second, and between the first and third equations, gives two expressions for the diagonal $r_{5}$, namely

$$
\begin{aligned}
& \beta_{1} r_{5}^{2}=\alpha_{1} \beta_{1} r_{1}^{2}+\alpha_{1} r_{2}^{2}-\alpha_{3}^{2} r_{3}^{2}, \\
& \alpha_{2} r_{5}^{2}=\beta_{2} r_{1}^{2}-\alpha_{2} \beta_{2} r_{2}^{2}+\beta_{4}^{2} r_{4}^{2} .
\end{aligned}
$$

Similarly, for the diagonal $r_{6}$,

$$
\begin{aligned}
& \beta_{4} r_{6}^{2}=\alpha_{4} r_{1}^{2}+\alpha_{4} \beta_{4} r_{4}^{2}-\alpha_{2}^{2} r_{2}^{2}, \\
& \alpha_{1} r_{6}^{2}=-\alpha_{1} \beta_{1} r_{1}^{2}+\beta_{3}^{2} r_{3}^{2}+\beta_{1} r_{4}^{2} .
\end{aligned}
$$

The elimination of $r_{5}{ }^{2}$ between the two equations of (8), or $r_{6}{ }^{2}$ between the two equations of (9), leads, after some reduction, to the equation

$$
\begin{aligned}
& \beta_{2}\left(\beta_{1} r_{1}^{2}+\alpha_{2} r_{2}^{2}\right)-\beta_{4}\left(\beta_{3} r_{3}^{2}+\alpha_{4} r_{4}^{2}\right)=0, \quad \text { or } \\
& \alpha_{1}\left(\beta_{1} r_{1}^{2}+\alpha_{2} r_{2}^{2}\right)-\alpha_{3}\left(\beta_{3} r_{3}^{2}+\alpha_{4} r_{4}^{2}\right)=0,
\end{aligned}
$$

since the determinant $\beta_{2} \alpha_{3}-\alpha_{1} \beta_{4}$ is zero.

Since $\alpha_{3}$ and $\alpha_{4}$ are expressible in terms of $\alpha_{1}$ and $\alpha_{2}$ by means of (7), equation (10) is a relation between the six quantities $r_{1}, r_{2}, r_{3}, r_{4}, \alpha_{1}, \alpha_{2}$. Regarding the four sides and $\alpha_{1}$ as given, this relation then determines $\alpha_{2}$, and 
then $\alpha_{3}$ and $\alpha_{4}$ by means of equations (7). For this purpose equation (10) is most simply expressed as a cubic in $\beta_{2}$, namely,

$$
\begin{aligned}
{\left[\alpha_{1}^{2} r_{2}^{2}-\beta_{1}{ }^{2} r_{4}^{2}\right] \beta_{2}{ }^{3}+\left[\alpha_{1}^{2} \beta_{1}\left(r_{1}^{2}-r_{3}^{2}\right)+\right.} & \left.\alpha_{1}^{2} r_{2}^{2}-\alpha_{1} \beta_{1} r_{4}^{2}\right] \beta_{2}^{2} \\
& -\left[\alpha_{1}^{2}\left(\alpha_{1}+\beta_{1}\right) r_{3}^{2}\right] \beta_{2}-\alpha_{1}{ }^{3} r_{3}^{2}=0 .
\end{aligned}
$$

Thus if the four sides $r_{1}, \cdots, r_{4}$ are given, there may be three quadrilaterals which have the same value of $\alpha_{1}$, but when a choice of these three has been made, $r_{5}^{2}$ and $r_{6}^{2}$ are simply computed by means of (8) and (9).

7. The resultant acceleration. The resultant acceleration of each of the particles $m_{1}, m_{2}, m_{3}$, and $m_{4}$ in turn due to the attraction of the other three particles is

$$
\begin{array}{ll}
\text { of } m_{1}, & \frac{m_{2}}{r_{1}{ }^{3}} r_{1} a_{1}+\frac{m_{3}}{r_{5}{ }^{3}} I_{13}-\frac{m_{4}}{r_{4}{ }^{3}} r_{4} a_{4}=A_{1}, \\
\text { of } m_{2}, & \frac{m_{3}}{r_{2}{ }^{3}} r_{2} a_{2}+\frac{m_{4}}{r_{6}{ }^{3}} I_{24}-\frac{m_{1}}{r_{1}{ }^{3}} r_{1} a_{1}=A_{2}, \\
\text { of } m_{3}, & \frac{m_{4}}{r_{3}{ }^{3}} r_{3} a_{3}+\frac{m_{1}}{r_{5}{ }^{3}} I_{31}-\frac{m_{2}}{r_{2}{ }^{3}} r_{2} a_{2}=A_{3}, \\
\text { of } m_{4}, & \frac{m_{1}}{r_{4}{ }^{3}} r_{4} a_{4}+\frac{m_{2}}{r_{6}{ }^{3}} I_{42}-\frac{m_{3}}{r_{3}{ }^{3}} r_{3} a_{3}=A_{4} .
\end{array}
$$

On substituting the values of $I_{i j}$ from (4) and using the notation

these expressions become

$$
\frac{1}{r_{i}^{3}}=R_{i}
$$

$$
\begin{aligned}
& A_{1}=\frac{1}{\alpha_{2}}\left[\left(\alpha_{2} R_{1} m_{2}+\beta_{2} R_{5} m_{3}\right) r_{1} a_{1}+\left(\beta_{4} R_{5} m_{3}-\alpha_{2} R_{4} m_{4}\right) r_{4} a_{4}\right], \\
& A_{2}=\frac{1}{\alpha_{3}}\left[\left(\alpha_{3} R_{2} m_{3}+\beta_{3} R_{6} m_{4}\right) r_{2} a_{2}+\left(\beta_{1} R_{6} m_{4}-\alpha_{3} R_{1} m_{1}\right) r_{1} a_{1}\right] \\
& A_{3}=\frac{1}{\alpha_{4}}\left[\left(\alpha_{4} R_{3} m_{4}+\beta_{4} R_{5} m_{1}\right) r_{3} a_{3}+\left(\beta_{2} R_{5} m_{1}-\alpha_{4} R_{2} m_{2}\right) r_{2} a_{2}\right], \\
& A_{4}=\frac{1}{\alpha_{1}}\left[\left(\alpha_{1} R_{4} m_{1}+\beta_{1} R_{6} m_{2}\right) r_{4} a_{4}+\left(\beta_{3} R_{6} m_{2}-\alpha_{1} R_{3} m_{3}\right) r_{3} a_{3}\right] .
\end{aligned}
$$

In order that the resulting acceleration on each particle may pass through the center of gravity of the system and be proportional to the distance of the particle from the center of gravity, it is necessary that

$$
A_{1}: A_{2}: A_{3}: A_{4}:: G_{1}: G_{2}: G_{3}: G_{4} \text {. }
$$


Let the factor of proportionality be $M R_{0}$, so that

$$
A_{i}-M R_{0} G_{i}=0 .
$$

Furthermore, let

$$
R_{i}-R_{0}=S_{i} .
$$

Then, on multiplying equations (6) by $R_{0}$ and subtracting from the corresponding equation of (12), it is found that the conditions which are necessary for a permanent configuration are

$$
\begin{aligned}
& \left(\alpha_{2} S_{1} m_{2}+\beta_{2} S_{5} m_{3}\right) r_{1} a_{1}+\left(\beta_{4} S_{5} m_{3}-\alpha_{2} S_{4} m_{4}\right) r_{4} a_{4}=0, \\
& \left(\alpha_{3} S_{2} m_{3}+\beta_{3} S_{6} m_{4}\right) r_{2} a_{2}+\left(\beta_{1} S_{6} m_{4}-\alpha_{3} S_{1} m_{1}\right) r_{1} a_{1}=0, \\
& \left(\alpha_{4} S_{3} m_{4}+\beta_{4} S_{5} m_{1}\right) r_{3} a_{3}+\left(\beta_{2} S_{5} m_{1}-\alpha_{4} S_{2} m_{2}\right) r_{2} a_{2}=0, \\
& \left(\alpha_{1} S_{4} m_{1}+\beta_{1} S_{6} m_{2}\right) r_{4} a_{4}+\left(\beta_{3} S_{6} m_{2}-\alpha_{1} S_{3} m_{3}\right) r_{3} a_{3}=0 .
\end{aligned}
$$

Of course the masses must be positive and, since

$$
M R_{0}=\frac{M}{r_{0}^{3}}=\omega^{2},
$$

$R_{0}$ must be positive if the forces are attractive.

8. The equations of condition. If the vectors $\boldsymbol{a}_{1}, \boldsymbol{a}_{2}, \boldsymbol{a}_{3}$, and $\mathbf{a}_{4}$ are noncollinear, it is necessary that all of the coefficients in equations (14) vanish, and since, by (7),

$$
\frac{\beta_{i+1}}{\alpha_{i-1}}=-\frac{\alpha_{i}}{\beta_{i}} \quad(i=1,2,3,4)
$$

(circular permutation of the subscripts), this requires that

$$
\begin{array}{ll}
S_{1} \alpha_{2} m_{2}+S_{5} \beta_{2} m_{3}=0, & S_{6} \alpha_{2} m_{2}+S_{3} \beta_{2} m_{3}=0, \\
S_{2} \alpha_{3} m_{3}+S_{6} \beta_{3} m_{4}=0, & S_{5} \alpha_{3} m_{3}+S_{4} \beta_{3} m_{4}=0, \\
S_{3} \alpha_{4} m_{4}+S_{5} \beta_{4} m_{1}=0, & S_{6} \alpha_{4} m_{4}+S_{1} \beta_{4} m_{1}=0, \\
S_{4} \alpha_{1} m_{1}+S_{6} \beta_{1} m_{2}=0, & S_{5} \alpha_{1} m_{1}+S_{2} \beta_{1} m_{2}=0 .
\end{array}
$$

In order to facilitate comparison, the equations in the second column have been circularly permuted once, that is, the last equation as derived from (14) has been placed first.

These equations are linear and homogeneous in the masses. A comparison of the first equations in each column shows that, since the determinant must vanish,

$$
S_{1} S_{3}=S_{5} S_{6}
$$


and from the other equations, taken in pairs, it is seen to be necessary that

$$
S_{1} S_{3}=S_{2} S_{4}=S_{6} S_{6} .
$$

If these conditions, equations (16), are satisfied, the equations in the second column of (15) will be satisfied if the equations in the first column are satisfied.

The determinant of the equations in the first column is easily found to be

$$
\Delta=S_{1} S_{2} S_{3} S_{4} \alpha_{1} \alpha_{2} \alpha_{3} \alpha_{4}-S_{5}^{2} S_{6}^{2} \beta_{1} \beta_{2} \beta_{3} \beta_{4},
$$

which, by virtue of equations (16), reduces to

$$
\Delta=S_{1} S_{2} S_{3} S_{4}\left(\alpha_{1} \alpha_{2} \alpha_{3} \alpha_{4}-\beta_{1} \beta_{2} \beta_{3} \beta_{4}\right) .
$$

From the first and third of equations (7) it is seen that

$$
\begin{aligned}
& \alpha_{1} \alpha_{2}=-\beta_{2} \beta_{3}, \\
& \alpha_{3} \alpha_{4}=-\beta_{4} \beta_{1} .
\end{aligned}
$$

Hence

$$
\alpha_{1} \alpha_{2} \alpha_{3} \alpha_{4}=\beta_{1} \beta_{2} \beta_{3} \beta_{4},
$$

and the determinant vanishes, if equations (16) are satisfied. Three of the masses can then be determined in terms of the fourth; for example, from (15)

$$
m_{2}=-\frac{\alpha_{1}}{\beta_{1}} \frac{S_{4}}{S_{6}} m_{1}, \quad m_{3}=-\frac{\beta_{3}}{\beta_{1}} \frac{S_{1}}{S_{2}} m_{1}, \quad m_{4}=+\frac{\alpha_{3}}{\beta_{1}} \frac{S_{1}}{S_{6}} m_{1} .
$$

9. The necessary condition. In order that the problem may admit a solution other than the straight line solution; it is necessary that

or

$$
S_{1} S_{3}=S_{2} S_{4}=S_{5} S_{6},
$$

$$
\begin{aligned}
\left(R_{1}-R_{0}\right)\left(R_{3}-R_{0}\right) & =\dot{(}\left(R_{2}-R_{0}\right)\left(R_{4}-R_{0}\right) \\
& =\left(R_{5}-R_{0}\right)\left(R_{6}-R_{0}\right) .
\end{aligned}
$$

From these equations it is found that

$$
\begin{aligned}
R_{0} & =\frac{R_{1} R_{3}-R_{2} R_{4}}{R_{1}+R_{3}-R_{2}-R_{4}}=\frac{R_{2} R_{4}-R_{5} R_{6}}{R_{2}+R_{4}-R_{5}-R_{6}} \\
& =\frac{R_{6} R_{6}-R_{1} R_{3}}{R_{5}+R_{6}-R_{1}-R_{3}}
\end{aligned}
$$

and therefore 


$$
\begin{aligned}
S_{1}=R_{1}-R_{0}= & \frac{\left(R_{1}-R_{2}\right)\left(R_{1}-R_{4}\right)}{R_{1}+R_{3}-R_{2}-R_{4}}=\frac{\left(R_{1}-R_{5}\right)\left(R_{1}-R_{6}\right)}{R_{1}+R_{3}-R_{5}-R_{6}}, \\
S_{2}=R_{2}-R_{0}= & \frac{\left(R_{2}-R_{1}\right)\left(R_{3}-R_{2}\right)}{R_{1}+R_{3}-R_{2}-R_{4}}=\frac{\left(R_{2}-R_{5}\right)\left(R_{2}-R_{6}\right)}{R_{2}+R_{4}-R_{6}-R_{6}}, \\
S_{3}=R_{3}-R_{0}= & \frac{\left(R_{3}-R_{2}\right)\left(R_{3}-R_{4}\right)}{R_{1}+R_{3}-R_{2}-R_{4}}=\frac{\left(R_{3}-R_{5}\right)\left(R_{3}-R_{6}\right)}{R_{1}+R_{3}-R_{6}-R_{6}}, \\
S_{4}=R_{4}-R_{0}= & \frac{\left(R_{3}-R_{4}\right)\left(R_{4}-R_{1}\right)}{R_{1}+R_{3}-R_{2}-R_{4}}=\frac{\left(R_{4}-R_{5}\right)\left(R_{4}-R_{6}\right)}{R_{2}+R_{4}-R_{5}-R_{6}}, \\
S_{5}=R_{5}-R_{0}= & \frac{\left(R_{1}-R_{5}\right)\left(R_{5}-R_{3}\right)}{R_{1}+R_{3}-R_{5}-R_{6}}=\frac{\left(R_{2}-R_{5}\right)\left(R_{5}-R_{4}\right)}{R_{2}+R_{4}-R_{5}-R_{6}}, \\
S_{6}=R_{6}-R_{0}= & \frac{\left(R_{1}-R_{6}\right)\left(R_{6}-R_{3}\right)}{R_{1}+R_{3}-R_{5}-R_{6}}=\frac{\left(R_{2}-R_{6}\right)\left(R_{6}-R_{4}\right)}{R_{2}+R_{4}-R_{5}-R_{6}} .
\end{aligned}
$$

From these expressions for $R_{0}$ it is seen that if two pairs of opposite sides are given, $R_{0}$ is determined uniquely except when the members of one pair are equal respectively to the members of the other pair. Suppose $r_{1}, r_{2}, r_{3}$, and $r_{4}$ are given and that the two members of the pair $r_{1}, r_{3}$ are not equal to the two members of the pair $r_{2}, r_{4}$. Then

$$
R_{0}=R_{1} R_{3}-R_{2} R_{4} / R_{1}+R_{3}-R_{2}-R_{4},
$$

and, automatically,

$$
S_{1} S_{3}=S_{2} S_{4}=\lambda,
$$

where $\lambda$ is some definite number. It is still necessary to determine the members of the other pair $r_{5}, r_{6}$, which may be called the diagonals, so that also

$$
S_{5} S_{6}=\lambda \text {. }
$$

For this purpose the shape of the quadrilateral is available.

Before going into this, however, it is desirable to take up the exceptional case first, the case in which equality exists between the members of the two given pairs of sides.

10. Particular case, $r_{1}=r_{2}, r_{3}=r_{4}$. In the particular case in which $r_{1}=r_{2}$ and $r_{3}=r_{4}$ it can be assumed that $r_{1} \geqq r_{3}$, as this is merely a matter of notation. The relation

$$
S_{1} S_{3}=S_{2} S_{4}
$$

is satisfied whatever $R_{0}$ may be. Consequently $R_{0}$ can be chosen so that

$$
S_{1} S_{3}=S_{2} S_{4}=S_{5} S_{6},
$$

that is, by using the second or third form of $R_{0}$ in equation (18). 
It is seen from Fig. 1 that, for this case,

and

$$
r_{2} \alpha_{2}=-r_{1} \beta_{1}
$$

so that

$$
r_{3} \alpha_{3}=-r_{4} \beta_{4}
$$

$$
\alpha_{2}=-\beta_{1}, \quad \alpha_{3}=-\beta_{4}, \quad \beta_{2}=-\alpha_{1}, \quad \beta_{3}=-\alpha_{4} .
$$

Then, by means of equations ( 7$)$, it is found that

$$
\begin{array}{ll}
\alpha_{2}=1-\alpha_{1}, & \beta_{2}=-\alpha_{1}, \\
\alpha_{3}=2-\alpha_{1}, & \beta_{3}=1-\alpha_{1}, \\
\alpha_{4}=\alpha_{1}-1, & \beta_{4}=\alpha_{1}-2,
\end{array}
$$

and all of the $\alpha$ 's and $\beta$ 's are expressed simply in terms of $\alpha_{1}$.

The expressions for the masses become

$$
\begin{aligned}
& m_{2}=-\frac{\alpha_{1}}{\beta_{1}} \frac{S_{4}}{S_{6}} m_{1}=-\frac{\alpha_{1}}{\beta_{1}} \frac{R_{5}-R_{3}}{R_{1}-R_{6}} m_{1}, \\
& m_{3}=m_{1}, \\
& m_{4}=+\frac{2-\alpha_{1}}{\alpha_{1}-1} \frac{S_{1}}{S_{6}} m_{1}=\frac{2-\alpha_{1}}{1-\alpha_{1}} \frac{R_{5}-R_{1}}{R_{6}-R_{3}} m_{1} ;
\end{aligned}
$$

and for the diagonals

$$
r_{5}{ }^{2}=\frac{\alpha_{1}{ }^{2}}{\alpha_{1}-1} r_{1}{ }^{2}-\frac{\left(2-\alpha_{1}\right)^{2}}{\alpha_{1}-1} r_{3}{ }^{2}, \quad r_{6}{ }^{2}=\left(1-\alpha_{1}\right)\left(r_{1}{ }^{2}-r_{3}{ }^{2}\right) .
$$

Under the assumption that $r_{1} \geqq r_{3}$, it is evident from the geometry that

$$
r_{1}-r_{3} \leqq r_{6} \leqq r_{1}+r_{3}
$$

and, if $\rho$ denotes the ratio of $r_{3}$ to $r_{1}$, it follows from the above expression for $r_{6}^{2}$ that

$$
-2 \rho /(1-\rho) \leqq \alpha_{1} \leqq 2 \rho /(1+\rho) \leqq+1 .
$$

It also follows that $\alpha_{1}=0$ when the sides $r_{3}$ and $r_{4}$ form a straight line; but this was already known from the definition of $\alpha_{1}$.

11. Conditions necessary for positive masses. In the particular case under discussion, $m_{3}$ is positive if $m_{1}$ is positive, which will be assumed. Starting with the maximum value of $\alpha_{1}$, namely

$$
\alpha_{1}=2 \rho /(1+\rho) \leqq 1,
$$

for which $r_{5}$ vanishes and $r_{6}=r_{1}-r_{3}$, the ratio $\alpha_{1} / \beta_{1}$ is negative until $\alpha_{1}$ vanishes and thereafter is positive, but the coefficient $\left(2-\alpha_{1}\right) /\left(1-\alpha_{1}\right)$ in $m_{4}$ 
is always positive. Changes of sign in the masses $m_{2}$ and $m_{4}$, considered as functions of $\alpha_{1}$ and $\rho$, occur for the following critical values (compare (21)):

$$
r_{5}=r_{3} \quad \text { for which } \rho=\frac{ \pm \alpha_{1}}{\left(3-3 \alpha_{1}+\alpha_{1}^{2}\right)^{1 / 2}}, m_{2}=0
$$

$$
r_{5}=r_{1} \text { for which } \rho=\frac{\left(1-\alpha_{1}+\alpha_{1}^{2}\right)^{1 / 2}}{2-\alpha_{1}}, \quad m_{4}=0 \text {, }
$$

$$
\alpha_{1}=0
$$$$
m_{2}=0 \text {, }
$$

(d)

$$
r_{6}=r_{3} \quad \text { for which } \alpha_{1}=\frac{1-2 \rho^{2}}{1-\rho^{2}}
$$$$
m_{4}=\infty \text {, }
$$

(e)

$$
r_{6}=r_{1} \text { for which } \alpha_{1}=\frac{-\rho^{2}}{1-\rho^{2}},
$$$$
m_{2}=\infty \text {. }
$$

The curves represented by these equations are shown in Fig. 2, in which $\alpha$ is the abscissa and $\rho$ is the ordinate. The area in which $m_{2}$ is positive is hatched horizontally. The area in which $m_{4}$ is positive is hatched obliquely. Consequently both $m_{2}$ and $m_{4}$ are positive in the area that is cross hatched. Any point in this cross hatched area leads to a real, positive solution of the problem, provided $R_{0}$ also is positive, as is actually the case. It is seen that there are two such areas that are not connected, and that one of these areas is sub-divided into two areas that are connected at the point $\alpha_{1}=+1 / 2$,

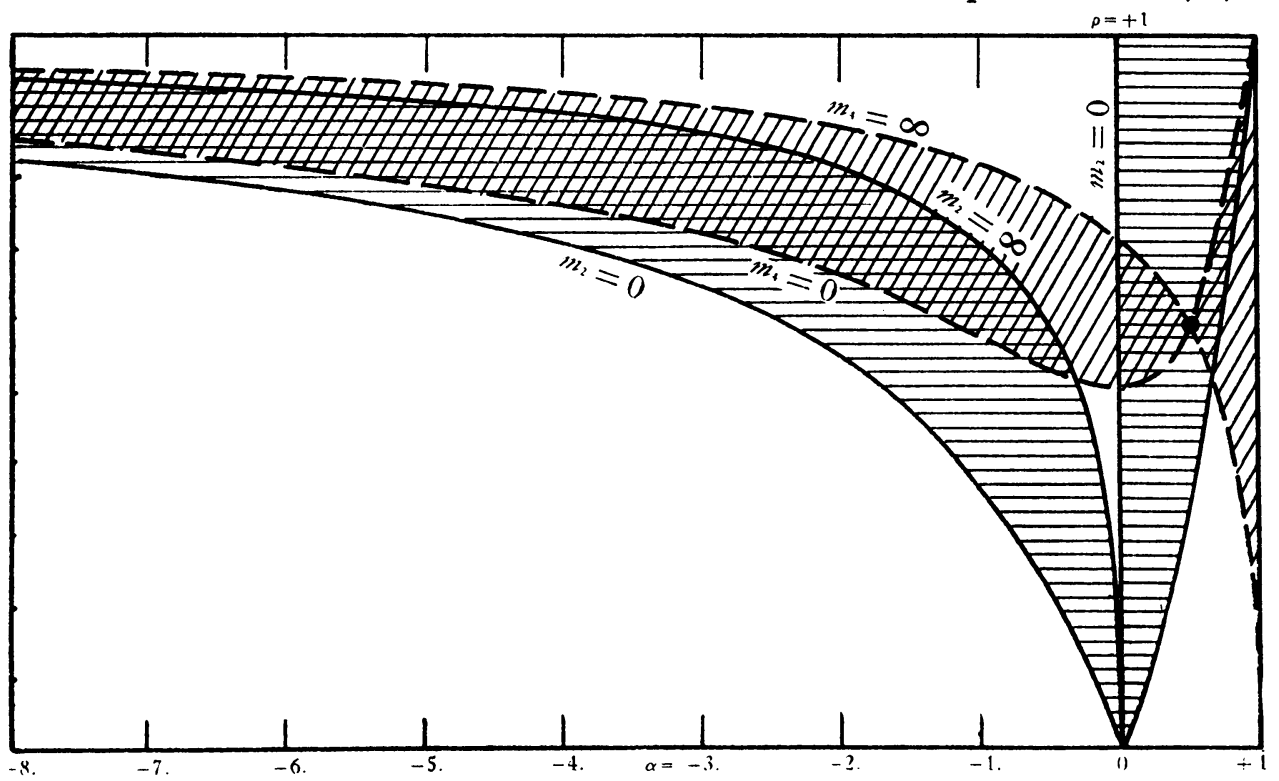

Fig. 2 
$\rho=1 / 3^{1 / 2}$. This point corresponds to the equilateral triangle in which $r_{1}=r_{2}$ $=r_{5}$, and since $r_{3}=r_{6}$, the fourth particle $m_{4}$ is at the center of the equilateral triangle. Three of the masses are arbitrary but equal, while the fourth is entirely arbitrary, and this is the only case in which the masses are not uniquely determined, if the quadrilateral is given.

The condition that $R_{0}=0$ is

$$
R_{1} R_{3}-R_{6} R_{6}=0,
$$

and this leads to the equation

The condition that $R_{0}=\infty$ is

$$
\rho^{2}=\frac{2 \alpha_{1}{ }^{2}-4 \alpha_{1}+3 \pm\left(3\left(2 \alpha_{1}-3\right)\left(2 \alpha_{1}-1\right)\right)^{1 / 2}}{2\left(2-\alpha_{1}\right)^{2}} .
$$

$$
R_{1}+R_{3}-R_{5}-R_{6}=0 .
$$

It can be expressed as an equation in $\rho$ and $\alpha_{1}$ but is too complicated to write down. Both of the curves $R_{0}=0$ and $R_{0}=\infty$ are shown in Fig. 3. The two

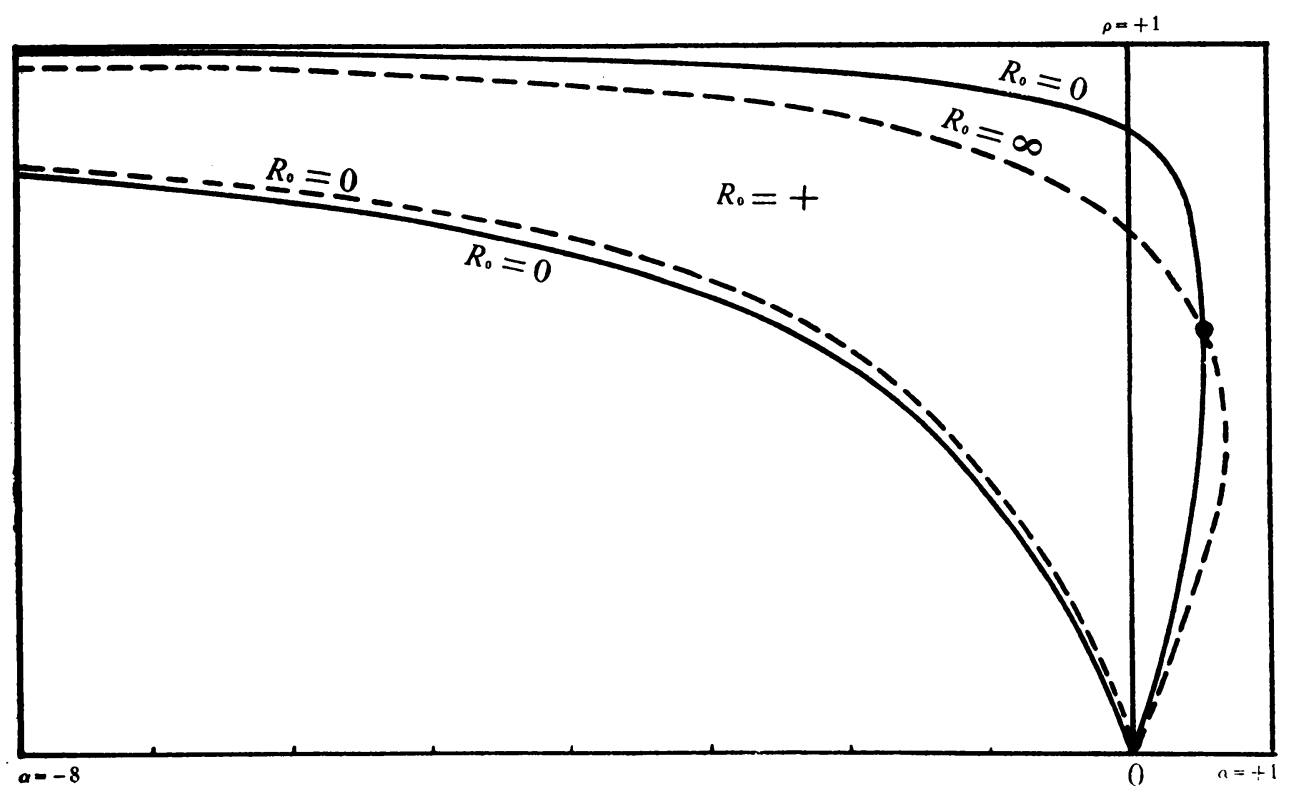

Fig. 3

curves intersect at the points 0,0 and.1/2,1/3/2, both of which are conspicuous in Fig. 2. A superposition of Figs. 2 and 3 shows that $R_{0}$ is positive everywhere within the cross hatched area of Fig. 2. There are therefore two groups of solutions for the special problem in which $r_{1}=r_{2}$. In the first group $\alpha_{1}$ is negative, and the quadrilateral is convex. In the second group 


$$
0 \leqq \alpha_{1} \leqq+1,
$$

and the quadrilateral is concave. The masses $m_{1}$ and $m_{3}$ are always equal, while $m_{2}$ and $m_{4}$ may have any positive values whatever.

12. Resumption of the general case. Aside from the exceptional case in which two pairs of adjacent sides are equal, $R_{0}$ is uniquely determined by the condition (\$9)

$$
S_{1} S_{3}=S_{2} S_{4}=\lambda \text {, }
$$

assuming that $r_{1}, r_{2}, r_{3}$ and $r_{4}$ are given. The shape of the quadrilateral, however, is still arbitrary, that is, $\alpha_{1}$ is still at our disposal. After $\alpha_{1}$ has been fixed $\alpha_{2}$ is determined by equation (11), which is a cubic in $\beta_{2}$, and then $\alpha_{3}$ and $\alpha_{4}$ by equations (7). Hence $r_{5}$ and $r_{6}$ can be thought of as functions of $\alpha_{1}$, and the point is to determine $\alpha_{1}$ so that

$$
S_{5} S_{6}=\lambda \text {. }
$$

From the first equation of (8) and the second equation of (9) are obtained and

$$
\alpha_{3} r_{3}= \pm\left(\alpha_{1} \beta_{1} r_{1}^{2}+\alpha_{1} r_{2}^{2}-\beta_{1} r_{5}^{2}\right)^{1 / 2}=Q_{1},
$$

$$
\beta_{3} r_{3}= \pm\left(\alpha_{1} r_{6}^{2}+\alpha_{1} \beta_{1} r_{1}^{2}-\beta_{1} r_{4}^{2}\right)^{1 / 2}=Q_{2} .
$$

Using the first of these equations, it is found that

$$
\begin{array}{lll}
\alpha_{2}=\frac{-r_{3}+Q_{1}}{\beta_{1} r_{3}+Q_{1}}, & \alpha_{3}=\frac{Q_{1}}{r_{3}}, & \alpha_{4}=\frac{\beta_{1} r_{3}}{\beta_{1} r_{3}+Q_{1}}, \\
\beta_{2}=\frac{-\alpha_{1} r_{3}}{\beta_{1} r_{3}+Q_{1}}, & \beta_{3}=\frac{-r_{3}+Q_{1}}{r_{3}}, & \beta_{4}=\frac{-Q_{1}}{\beta_{1} r_{3}+Q_{1}} ;
\end{array}
$$

and from the second equation,

$$
\begin{array}{lll}
\alpha_{2}=\frac{Q_{2}}{\alpha_{1} r_{3}+Q_{2}}, & \alpha_{3}=1+\frac{Q_{2}}{r_{3}}, & \alpha_{4}=\frac{\beta_{1} r_{3}}{\alpha_{1} r_{3}+Q_{2}}, \\
\beta_{2}=\frac{-\alpha_{1} r_{3}}{\alpha_{1} r_{3}+Q_{2}}, & \beta_{3}=\frac{Q_{2}}{r_{3}}, & \beta_{4}=-\frac{r_{3}+Q_{2}}{\alpha_{1} r_{3}+Q_{2}} .
\end{array}
$$

On substituting these results in the second equation of (8) and the first equation of (9), the two following equations are derived:

$$
\begin{aligned}
-\beta_{1} r_{5}^{4} & +\left[\alpha_{1}^{2} r_{1}^{2}+\alpha_{1}\left(-r_{1}^{2}+r_{2}^{2}-r_{3}^{2}+r_{4}^{2}\right)+\left(r_{3}^{2}-r_{4}^{2}\right)\right] r_{5}^{2} \\
& +\left[\alpha_{1}^{2} r_{1}^{2}\left(r_{3}^{2}-r_{4}^{2}\right)+\alpha_{1}\left(r_{3}^{2}-r_{4}^{2}\right)\left(r_{2}^{2}-r_{1}^{2}\right)\right] \\
& +\left[\alpha_{1}\left(r_{1}^{2}-r_{2}^{2}+r_{5}^{2}\right)-2 r_{5}^{2}\right] r_{3} Q_{1}=0,
\end{aligned}
$$

and 


$$
\begin{aligned}
+\alpha_{1} r_{6}{ }^{4} & +\left[\alpha_{1}^{2} r_{1}{ }^{2}+\alpha_{1}\left(-r_{1}{ }^{2}-r_{2}{ }^{2}+r_{3}{ }^{2}-r_{4}{ }^{2}\right)+r_{4}{ }^{2}\right] r_{6}{ }^{2} \\
& +\left[-\alpha_{1}{ }^{2}\left(r_{2}{ }^{2}-r_{3}^{2}\right) r_{1}{ }^{2}+\alpha_{1}\left(r_{1}{ }^{2}+r_{4}{ }^{2}\right)\left(r_{2}{ }^{2}-r_{3}{ }^{2}\right)-\left(r_{2}{ }^{2}-r_{3}{ }^{2}\right) r_{4}{ }^{2}\right] \\
& +\left[\alpha_{1}\left(r_{1}{ }^{2}-r_{4}{ }^{2}+r_{6}{ }^{2}\right)+\left(-r_{1}{ }^{2}+r_{4}{ }^{2}+r_{6}{ }^{2}\right)\right] r_{3} Q_{2}=0 .
\end{aligned}
$$

If rationalized the first would be an equation of the eighth degree in $r_{5}$ and the second of the eighth degree in $r_{6}$. These equations determine $r_{5}$ and $r_{6}$ as functions of $\alpha_{1}$ and the four sides $r_{1}, r_{2}, r_{3}$, and $r_{4}$. On substituting these values of $r_{5}$ and $r_{6}$ in the equation

$$
\left(\frac{1}{r_{i}^{3}}-\frac{1}{r_{0}{ }^{3}}\right)\left(\frac{1}{r_{6}{ }^{3}}-\frac{1}{r_{10}^{3}}\right)=\lambda
$$

there is derived an equation which determines $\alpha_{1}$. It is not practical to do this literally. One can determine $\alpha_{1}$ as accurately as may be desired from these equations by a series of approximations in numerical cases, but the process is laborious.

13. Convex and concave quadrilaterals. Suppose that pegs are placed at each of the four masses. A string is passed around them and drawn taut. Two cases are distinguishable:

I. The string touches all four pegs, and

(a) no three pegs are in a straight line;

(b) three pegs are in a straight line, but not four;

(c) four pegs are in a straight line.

II. The string touches only three pegs, the fourth being inside of the triangle formed by the other three.

If the conditions of Case $\mathrm{I}(\mathrm{a})$ or $\mathrm{I}(\mathrm{b})$ are satisfied, the quadrilateral will be called convex. Case $\mathrm{I}(\mathrm{c})$ is the straight line configuration with which we are not concerned. If the conditions of Case II are satisfied, the quadrilateral will be called concave.

In all cases at least three masses touch the string. Let these three masses in counterclockwise order be $m_{1}, m_{2}$, and $m_{3}$. If $m_{4}$ also lies on the string it is between $m_{3}$ and $m_{1}$ and the quadrilateral is convex. If it does not touch the string it lies inside of the triangle formed by $m_{1}, m_{2}$, and $m_{3}$, and the quadrilateral is concave. This convention as to the masses eliminates duplication of cases that differ essentially in notation only.

It is seen from Fig. 1 that the ratio

$$
\frac{\alpha_{1}}{\beta_{1}}=\frac{\alpha_{1}}{\alpha_{1}-1}
$$

is positive wherever the point $B$ on the line $L_{1}$ may be, provided it does not lie between $m_{1}$ and $m_{2}$, and in the interval $m_{1} m_{2}$ it is negative. In general, 
(24) for convex quadrilaterals, $\frac{\alpha_{1}}{\beta_{1}}>0, \frac{\alpha_{2}}{\beta_{2}}>0, \frac{\alpha_{3}}{\beta_{3}}>0, \frac{\alpha_{4}}{\beta_{4}}>0$;

and

$$
\text { for concave quadrilaterals, } \frac{\alpha_{1}}{\beta_{1}}<0, \frac{\alpha_{2}}{\beta_{2}}<0, \frac{\alpha_{3}}{\beta_{3}}>0, \frac{\alpha_{4}}{\beta_{4}}>0 \text {. }
$$

14. Admissible convex quadrilaterals. From equations (13), (15), and (16) one sees that in any solution of the problem

and

$$
\begin{aligned}
& m_{2}=-\frac{\alpha_{1}}{\beta_{1}} \frac{R_{4}-R_{0}}{R_{6}-R_{0}} m_{1}=-\frac{\alpha_{1}}{\beta_{1}} \frac{R_{5}-R_{0}}{R_{2}-R_{0}} m_{1}, \\
& m_{3}=-\frac{\alpha_{2}}{\beta_{2}} \frac{R_{1}-R_{0}}{R_{5}-R_{0}} m_{2}=-\frac{\alpha_{2}}{\beta_{2}} \frac{R_{6}-R_{0}}{R_{3}-R_{0}} m_{2}, \\
& m_{4}=-\frac{\alpha_{3}}{\beta_{3}} \frac{R_{2}-R_{0}}{R_{6}-R_{0}} m_{3}=-\frac{\alpha_{3}}{\beta_{3}} \frac{R_{5}-R_{0}}{R_{4}-R_{0}} m_{3}, \\
& m_{1}=-\frac{\alpha_{4}}{\beta_{4}} \frac{R_{3}-R_{0}}{R_{5}-R_{0}} m_{4}=-\frac{\alpha_{4}}{\beta_{4}} \frac{R_{6}-R_{0}}{R_{1}-R_{0}} m_{4}
\end{aligned}
$$

First hypothesis: $r_{1}>r_{0}$. From their definitions it follows, if $r_{1}>r_{0}$, that

$$
R_{1}<R_{0}
$$

and since for convex quadrilaterals $\alpha_{i} / \beta_{i}>0, i=1,2,3,4$, and since the masses are necessarily positive, it is found from equations (26) that

$$
R_{1}, R_{2}, R_{3}, R_{4}<R_{0}<R_{5}, R_{6}
$$

and therefore

$$
r_{1}, r_{2}, r_{3}, r_{4}>r_{0}>r_{5}, r_{6}
$$

that is, each of the four sides is greater than $r_{0}$, and each of the two diagonals is less than $r_{0}$. This is a geometric absurdity for a convex quadrilateral, as is easily proved. Hence there are no solutions of the problem in which $r_{1}>r_{0}$.

Second hypothesis: $r_{1} \leqq r_{0}$. This hypothesis merely reverses the inequalities of the first hypothesis, but includes the equality sign. Hence, in this case,

$$
r_{1}, r_{2}, r_{3}, r_{4} \leqq r_{0} \leqq r_{5}, r_{6}
$$

that is, each of the four sides is less than, or, at most, equal to $r_{0}$, and each of 
the diagonals is greater than $r_{0}$. Quadrilaterals of this type are geometrically possible. In order to show this, let $r_{0}$ and $r_{1}$ be given with

$$
r_{1}<r_{0}
$$

Draw $r_{1}$ as in Fig. 4, and let $m_{1}$ and $m_{2}$ be at its extremities. With $m_{1}$ and

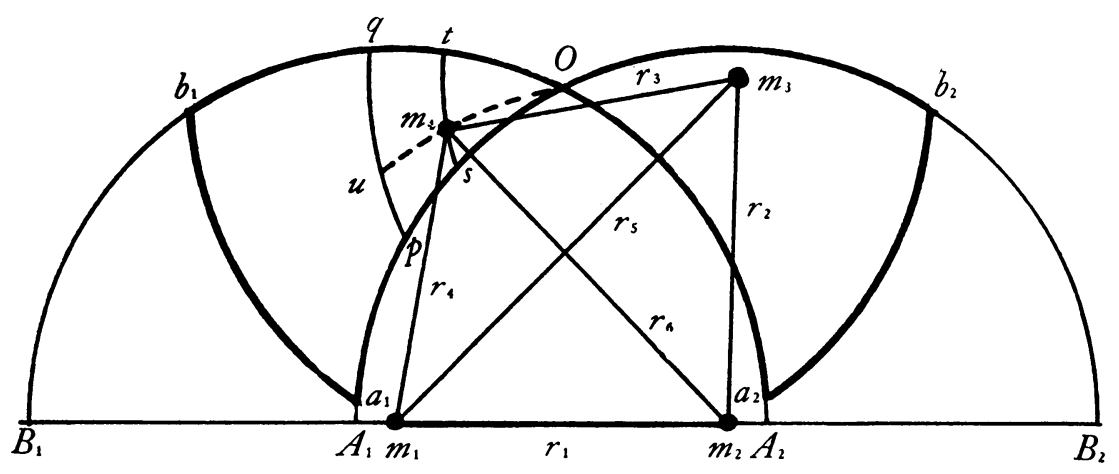

Fig. 4

$m_{2}$ as centers draw semicircles with the radius $r_{0}$, intersecting at the point $O$. With $O$ as a center draw the $\operatorname{arcs} a_{1} b_{1}$ and $a_{2} b_{2}$ also with the radius $r_{0}$. Since

$$
r_{5}>r_{0}>r_{2}
$$

the mass $m_{3}$ lies outside of the semicircle $B_{1} O A_{2}$ and inside of the semicircle $A_{1} O B_{2}$; that is, it lies inside of the area $O A_{2} B_{2}$. Likewise, since

$$
r_{6}>r_{0}>r_{4}
$$

the mass $m_{4}$ lies outside of the semicircle $A_{1} O B_{2}$ and inside of the semicircle $B_{1} O A_{2}$; that is, it lies inside of the area $O A_{1} B_{1}$. The possibilities are further restricted by the fact that $r_{3}$, which is the line joining $m_{3}$ and $m_{4}$, also is less than $r_{0}$. Hence $m_{3}$ must lie inside of the area $O a_{2} b_{2}$, and $m_{4}$ must lie inside of the area $O b_{1} a_{1}$; and the distance between $m_{3}$ and $m_{4}$ must be less than $r_{0}$.

A quadrilateral will be called an admissible quadrilateral if, for properly chosen masses, it can form a permanent configuration. With this definition we can state the following theorem:

THEOREM. For every point $m_{3}$ in the region $\mathrm{Oa}_{2} b_{2}$ there exists one and only one point $m_{4}$ in the region $O b_{1} a_{1}$ which together with the points $m_{1}$ and $m_{2}$ forms an admissible convex quadrilateral; and for every point $m_{4}$ in the region $O b_{1} a_{1}$ there exists one and only one point $m_{3}$ in the region $\mathrm{Oa}_{2} b_{2}$ which together with the points $m_{1}$ and $m_{2}$ forms an admissible convex quadrilateral. No such points exist outside of the areas $O b_{1} a_{1}$ and $O a_{2} b_{2}$. 
Let $m_{3}$ be taken anywhere within the area $O a_{2} b_{2}$. With $m_{3}$ as a center and a radius $r_{0}$ describe the arc $p q$ intersecting the $\operatorname{arcs} O a_{1}$ and $O b_{1}$ in $p$ and $q$. Any point $m_{4}$ which lies in the area $O p q$ together with $m_{1}, m_{2}$, and $m_{3}$ will form a quadrilateral which satisfies the inequalities

$$
r_{1}, r_{2}, r_{3}, r_{4}<r_{0}<r_{5}, r_{6} .
$$

It remains to show that there is one and only one such point at which the equalities

$$
\left(R_{1}-R_{0}\right)\left(R_{3}-R_{0}\right)=\left(R_{2}-R_{0}\right)\left(R_{4}-R_{0}\right)=\left(R_{0}-R_{6}\right)\left(R_{0}-R_{6}\right)
$$

also are satisfied. The quantities $R_{0}, R_{1}, R_{2}$, and $R_{5}$ are given by the assumed data.

All of the factors which occur in (28) are positive in the area $O p q$. With $m_{3}$ as a center and a radius $\rho$, such that

$$
\overline{O m_{3}} \leqq \rho \leqq r_{0},
$$

describe an arc of a circle which cuts $O p$ at the point $s$ and $O q$ at the point $t$. Imagine the point $m_{4}$ lying on this arc $s t$ and moving from $s$ to $t$. The factor $\left(R_{4}-R_{0}\right)$ is positive at $s$, decreases steadily, and vanishes at $t$, while the factor $\left(R_{0}-R_{6}\right)$ vanishes at $s$, and, increasing steadily, is positive at $t$. Hence there exists one and only one point $p$ on st at which the equality

$$
\left(R_{2}-R_{0}\right)\left(R_{4}-R_{0}\right)=\left(R_{0}-R_{6}\right)\left(R_{0}-R_{6}\right)=\lambda(\rho)
$$

is satisfied. This is true for every value of $\rho$, and the locus of $p$ as $\rho$ increases is a certain curve $C$ which passes through the point $O$ and cuts the arc $p q$ in some point $u$. It is evident that $\lambda(\rho)$ vanishes at $O$. Its derivative with respect to $\rho$ is

$$
\frac{d \lambda}{d \rho}=\left(R_{2}-R_{0}\right) \frac{d R_{4}}{d \rho}=-\left(R_{0}-R_{6}\right) \frac{d R_{6}}{d \rho}
$$

Since $R_{i}=1 / r_{i}{ }^{3}$,

and therefore

$$
\frac{d R_{4}}{d \rho}>0 \text { and } \frac{d R_{6}}{d \rho}<0
$$

$$
\frac{d \lambda}{d \rho}>0
$$

as the point $p$ moves along the curve $C_{1}$ at $O$. Regarding $r_{4}$ and $r_{6}$ as bipolar coördinates of the point $p$, it is seen that $d r_{4}$ and $d r_{6}$ cannot both vanish, since the point $p$ does not lie in the line which passes through $m_{1}$ and $m_{2}$. 
Therefore $d R_{4}$ and $d R_{6}$ cannot vanish simultaneously, and since $\left(R_{2}-R_{0}\right)$ and $\left(R_{0}-R_{5}\right)$ are constants, it is seen from (29) that neither can vanish, and therefore $d \lambda / d \rho$ is always positive. Hence the value of $\lambda$ increases steadily from zero at $O$ to some positive value at $u$.

On the other hand the value of $\left(R_{1}-R_{0}\right)\left(R_{3}-R_{0}\right)$ is positive at $O$, decreases steadily, and vanishes at $u$. Hence, for a given $m_{3}$, there exists one and only one point $m_{4}$ on the curve $C$, and therefore within the area $O p q$, at which the equalities

$$
\left(R_{1}-R_{0}\right)\left(R_{3}-R_{0}\right)=\left(R_{2}-R_{0}\right)\left(R_{4}-R_{0}\right)=\left(R_{0}-R_{5}\right)\left(R_{0}-R_{6}\right)
$$

are satisfied.

The first half of the theorem as stated is therefore established; and the second half follows from symmetry.

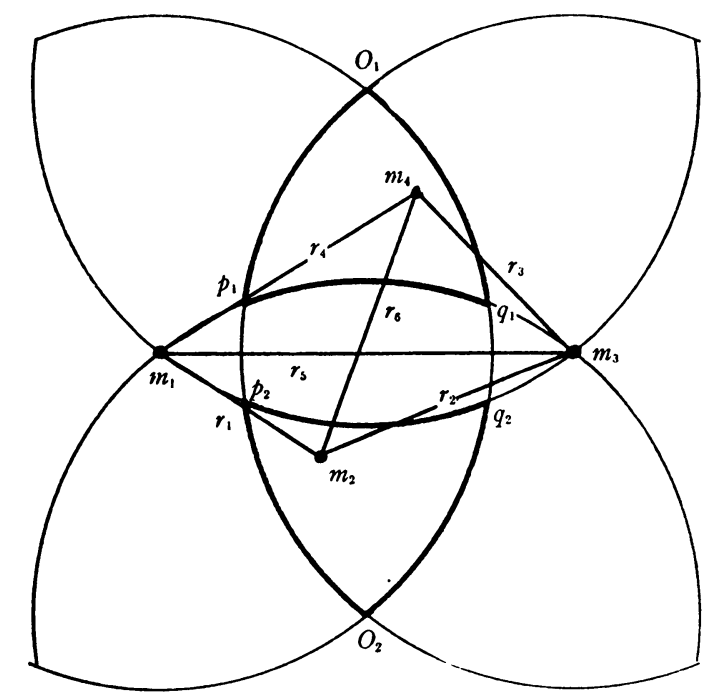

Fig. 5

15. The ratio of the diagonals. Instead of drawing the diagram with $r_{1}$ and $r_{0}$ as the fundamental lines, let $r_{5}$ and $r_{0}$ be used $\left(r_{5}>r_{0}\right)$. In Fig. 5, let $m_{1}$ and $m_{3}$ be the end points of $r_{5}$. With each of these points as a center draw circles of radius $r_{0}$ intersecting at the points $O_{1}$ and $O_{2}$. With the points $O_{1}$ and $O_{2}$ as centers draw two more circles with the radius $r_{0}$. These last two circles pass through $m_{1}$ and $m_{3}$ and intersect the $\operatorname{arcs} O_{1} O_{2}$ in the points $p_{1}, q_{1} ; p_{2}$, and $q_{2}$.

Since for all admissible quadrilaterals

$$
r_{1}, r_{2}, r_{3}, r_{4} \leqq r_{0} \leqq r_{5}, r_{6},
$$

it is seen that $m_{4}$ lies in the area $O_{1} p_{1} q_{1}$, and $m_{3}$ lies in the area $O_{2} p_{2} q_{2}$, and it 
can be shown, just as before, that for every $m_{4}$ in the area $O_{1} p_{1} q_{1}$ there exists one and only one $m_{2}$ in the area $O_{2} p_{2} q_{2}$ which, together with $m_{1}, m_{3}$, and $m_{4}$, forms an admissible quadrilateral. Similarly, for each $m_{2}$ in $\mathrm{O}_{2} p_{2} q_{2}$ there is one and only one $m_{4}$ in the area $O_{1} p_{1} q_{1}$.

It is evident that if $r_{0}$ is kept fixed, and $r_{1}$ is increased, the areas $O_{i} p_{i} q_{i}$ shrink and for a certain value of $r_{1}$ are reduced to two points. For larger values of $r_{1}$ admissible convex quadrilaterals do not exist.

From Fig. 5 is obtained

$$
\left(\frac{r_{5}}{2}\right)^{2}+\left(\frac{O_{1} O_{2}}{2}\right)^{2}=r_{0}^{2}
$$

But since $\overline{O_{1} O_{2}} \geqq r_{6} \geqq r_{0}$, it follows that

$$
r_{5} \leqq 3^{1 / 2} r_{0}, \quad \text { and } \quad r_{6} \leqq 3^{1 / 2} r_{0}
$$

therefore

$$
r_{5} \leqq 3^{1 / 2} r_{6}, \quad \text { and } \quad r_{6} \leqq 3^{1 / 2} r_{5}
$$

Whence the

THEOREM. The ratio of the diagonals of an admissible convex quadrilateral lies between $1 / 3^{1 / 2}$ and $3^{1 / 2}$.

This theorem is a generalization of Longley's theorem for the rhombus.

16. Limitations on the interior angles. There is a corresponding limitation on the magnitudes of the interior angles of an admissible convex quadrilateral. It is evident from Fig. 5 that the interior angle at $m_{1}, \angle m_{1}$, is less than the angle $O_{1} m_{1} O_{2}$; and that the maximum value of this latter angle is had at the limit $r_{1}=r_{0}$, in which case it is $120^{\circ}$. Hence

$$
\angle m_{1} \leqq 120^{\circ} \text {. }
$$

From Fig. 4 it is also evident that the $\angle m_{1}$ is greater than the angle $O m_{1} m_{2}$, and that the minimum value of $\angle O m_{1} m_{2}$ is had for the limiting value $r_{1}=r_{0}$ in which case it is $60^{\circ}$. Combining these two results, we have

$$
60^{\circ} \leqq \angle m_{1} \leqq 120^{\circ} \text {. }
$$

The same inequality holds obviously for $\angle m_{2}$. On interchanging the rôle of $m_{4}$ and $m_{1}$, and $m_{3}$ and $m_{2}$, it is seen that the same inequality holds for all of the interior angles.

THEOREM. Each of the interior angles of an admissible convex quadrilateral lies between $60^{\circ}$ and $120^{\circ}$.

By a similar method, using Fig. 5, it can be shown that we have the 
THEOREM. In any admissible convex quadrilateral the diagonals divide each of the interior angles into two angles each of which is less than $60^{\circ}$.

17. Admissible concave quadrilaterals. If $r_{1}<r_{0}$, it is found from equations (25) and (26) that

$$
r_{3}, r_{4}, r_{6}>r_{0}>r_{1}, r_{2}, r_{5}
$$

which is geometrically impossible for a concave quadrilateral.

If $r_{1} \geqq r_{0}$, the inequality signs are reversed and the equality sign is added. Hence a necessary condition for admissible concave quadrilaterals is that

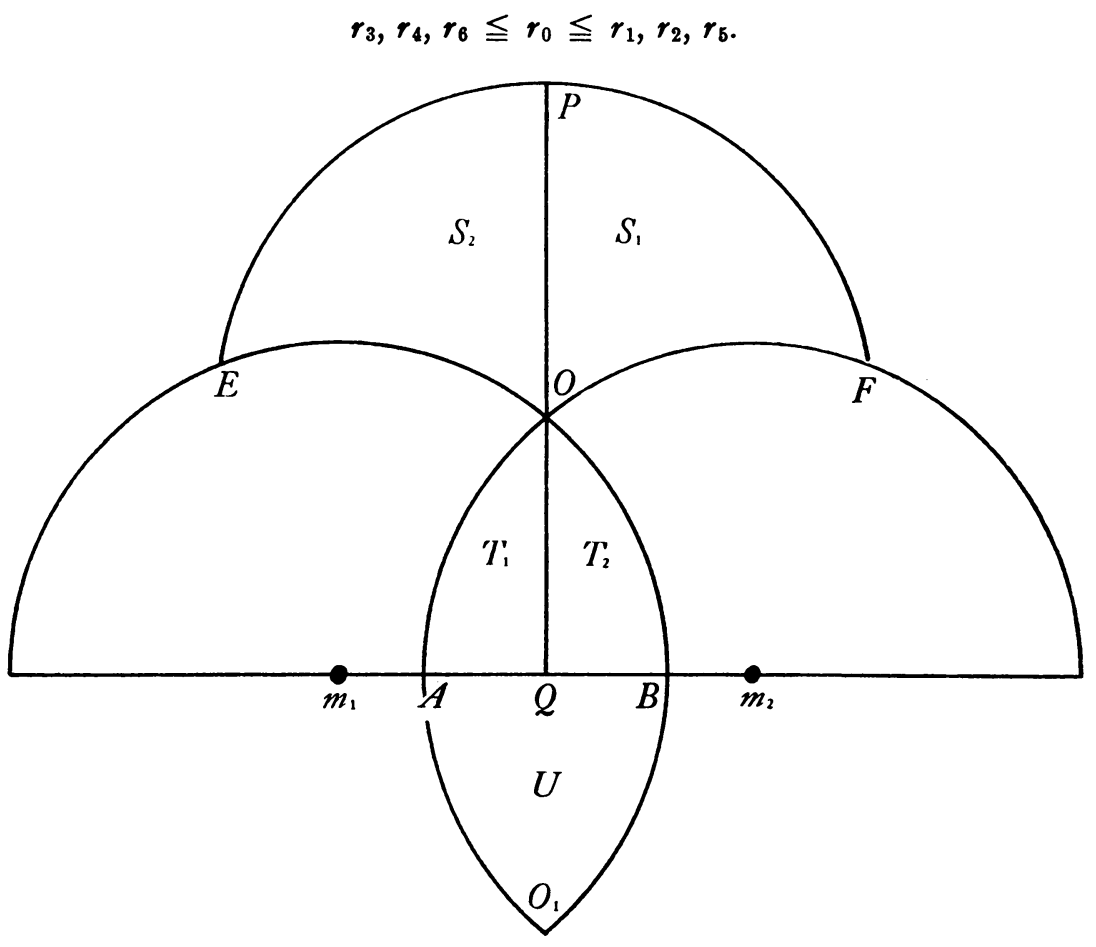

Fig. 6

The possibility of quadrilaterals of this type is shown in Fig. 6. Let $\overline{m_{1} m_{2}}$ be $r_{1}$. With $m_{1}$ and $m_{2}$ as centers draw circles of radius $r_{0}$. Since $r_{4}+r_{6} \geqq r_{1} \geqq r_{0}$ and $r_{4}, r_{6} \leqq r_{0}$, it is seen that

$$
r_{0} \leqq r_{1} \leqq 2 r_{0} .
$$

Let these two circles intersect in the points $O$ and $O_{1}$. With $O$ as a center and a radius equal to $r_{0}$ draw the arc $E P F$.

On account of the inequalities (30) and the adopted conventions ( $\$ 13)$ 
it is evident that $m_{4}$ must lie in the region $T_{1}$ or $T_{2}$, and $m_{3}$ must lie in $S_{1}$ or $S_{2}$. An argument similar to that used in $\$ 14$ shows that if $m_{4}$ is any assigned point in $T_{1}+T_{2}$ there exists one and only one point $m_{3}$ in the region $S_{1}+S_{3}$; but for any assigned point $m_{3}$ in the region $S_{1}+S_{3}$ there exists one and only one point $m_{4}$ in the region $T_{1}+T_{2}+U$, at which the equalities

$$
\begin{aligned}
\left(R_{0}-R_{1}\right)\left(R_{3}-R_{0}\right) & =\left(R_{0}-R_{2}\right)\left(R_{4}-R_{0}\right) \\
& =\left(R_{0}-R_{5}\right)\left(R_{6}-R_{0}\right)
\end{aligned}
$$

are satisfied. All of the factors in (31) are positive if $m_{3}$ and $m_{4}$ lie in the assigned regions. One sees from the last equality, if $r_{2}<r_{5}$, that $r_{4}<r_{6}$, and therefore if $m_{4}$ lies in $T_{1}, m_{3}$ lies in $S_{1}$; and if $m_{4}$ lies in $T_{2}, m_{3}$ lies in $S_{2}$. Notwithstanding the fact that for all admissible concave quadrilaterals $m_{4}$ lies in $T_{1}+T_{2}$ and $m_{3}$ in $S_{1}+S_{2}$, it is not true that all quadrilaterals which satisfy this condition are concave. There exist convex quadrilaterals for which $m_{4}$ lies in $T_{1}+T_{2}$ and $m_{3}$ in $S_{1}+S_{2}$ for which the equalities are satisfied, but for all such convex quadrilaterals at least one of the masses is negative, as was shown in $\$ 14$. This means that the regions $S_{1}+S_{2}$ and $T_{1}+T_{2}$ are not sufficiently restricted. A plane concave quadrilateral cannot change in a continuous manner into a convex quadrilateral without passing through a configuration in which three of the corners lie in a straight line, and the curves on which this happens pass through the regions $T_{1}, T_{2}, S_{1}$, and $S_{2}$. Since the masses are all positive for the concave quadrilaterals and at least one of the masses negative for the convex quadrilaterals, it follows that at least one of the masses vanishes on the boundary.

There are three of these bounding loci:

(I) $m_{4}$ lies on the line $m_{1} m_{2}$,

(II) $m_{4}$ lies on the line $m_{1} m_{3}$,

(III) $m_{4}$ lies on the line $m_{2} m_{3}$.

The two dynamical equations (31) must be satisfied in all cases, but the geometric equations (8) and (9) take different forms in the different cases.

Case I. $m_{4}$ lies between $m_{1}$ and $m_{2}$. One finds readily under this condition that

$$
\begin{gathered}
\alpha_{1}=\frac{r_{4}}{r_{1}}, \quad \alpha_{2}=0, \quad \alpha_{3}=+1, \\
\alpha_{4}=1-\frac{r_{1}}{r_{4}}=-\frac{r_{6}}{r_{4}},
\end{gathered}
$$

and the geometric equations (8) and (9) reduce to the two equations 


$$
\begin{aligned}
r_{1} & =r_{4}+r_{6} \\
r_{1} r_{3}^{2} & =r_{4} r_{2}^{2}+r_{6} r_{5}^{2}-r_{1} r_{4} r_{6} .
\end{aligned}
$$

Thus between the five quantities $r_{2}, r_{3}, r_{4}, r_{5}$, and $r_{6}$ there exist four relations. One can imagine $r_{3}, r_{5}$, and $r_{6}$ eliminated between these four equations, leaving a single relation which defines the locus sought by means of the bipolar coördinates $r_{2}$ and $r_{4}$. Actually it is not practical to do this, and one must resort to methods of successive approximations in numerical cases in order to obtain points on the curves. It should be noticed that these curves depend upon the ratio $r_{0} / r_{1}$, and therefore cannot be drawn once for all.

It is easy to solve the equations if $m_{4}$ coincides with either of the points $A$ or $B$ of Fig. 6. The following are the results:

$$
\begin{aligned}
\text { at } A, \quad r_{2}=r_{3} & =r_{6}=r_{0}, \quad r_{4}=r_{1}-r_{0}, \\
r_{5}^{2} & =r_{1}^{2}-r_{1} r_{0}+r_{0}^{2} ; \\
\text { at } B, \quad r_{3}=r_{4} & =r_{5}=r_{0}, \quad r_{6}=r_{1}-r_{0}, \\
r_{2}^{2} & =r_{1}^{2}-r_{1} r_{0}+r_{0}^{2} .
\end{aligned}
$$

At the point $Q$, a fairly simple solution can be obtained, since

$$
r_{1}=2 r_{4}=2 r_{6}
$$

and the necessary equations reduce to

$$
\begin{aligned}
\left(R_{0}-R_{1}\right)\left(R_{3}-R_{0}\right) & =\left(R_{0}-R_{2}\right)\left(8 R_{1}-R_{0}\right), \\
r_{2}{ }^{2} & =r_{3}{ }^{2}+\frac{1}{4} r_{1}^{2} .
\end{aligned}
$$

Let $r_{3}=\lambda r_{1}$, so that $r_{2}^{2}=\left(\lambda^{2}+\frac{1}{4}\right) r_{1}^{2}$. Also, if one takes $\lambda^{-3}=a$ and $\left(\lambda^{2}+\frac{1}{4}\right)^{-3 / 2}$ $=b$, then

$$
R_{3}=a R_{1}, \quad R_{2}=b R_{1}
$$

and

$$
R_{1}=\frac{7+b-a}{8 b-a}-R_{0} .
$$

In order that the inequalities $r_{0}<r_{1}<2 r_{1}$ may hold it is readily found that

$$
3^{1 / 2}>2 \lambda>1 \text {. }
$$

The following table shows the values of the various ratios if $m_{4}$ is at the central point $Q$. 
Table I. Values of the Ratios at $Q$

$\begin{array}{lccc}\lambda & \frac{r_{1}}{r_{0}}=\frac{2 r_{4}}{r_{0}}=\frac{2 r_{6}}{r_{0}} & \frac{r_{3}}{r_{0}} & \frac{r_{2}}{r_{0}} \\ .8667 & 1.000 & .8667 & 1.000 \\ .85 & 1.014 & .8620 & 1.000 \\ .80 & 1.067 & .8536 & 1.006 \\ .75 & 1.126 & .8445 & 1.015 \\ .70 & 1.195 & .8365 & 1.028 \\ .65 & 1.281 & .8326 & 1.041 \\ .64 & 1.301 & .8326 & 1.056 \\ .63 & 1.322 & .8329 & 1.063 \\ .62 & 1.344 & .8333 & 1.070 \\ .61 & 1.370 & .8357 & 1.080 \\ .60 & 1.396 & .8376 & 1.090 \\ .59 & 1.425 & .8408 & 1.102 \\ .58 & 1.458 & .8456 & 1.116 \\ .57 & 1.492 & .8504 & 1.131 \\ .56 & 1.533 & .8584 & 1.151 \\ .55 & 1.578 & .8679 & 1.173 \\ .54 & 1.631 & .8807 & 1.200 \\ .53 & 1.696 & .8989 & 1.236 \\ .52 & 1.772 & .9214 & 1.278 \\ .51 & 1.869 & .9532 & 1.3314 \\ .50 & 2.000 & 1.0000 & 1.4141 \\ \text { erpolation } & 1.3 & .8325 & 1.055 \\ & 1.4 & .838 & 1.090 \\ & 1.5 & .852 & 1.134\end{array}$

The following table gives values of the ratios on this curve for $r_{1}=1.5 r_{0}$.

$\begin{array}{rccc}r_{2} / r_{0} & r_{3} / r_{0} & r_{4} / r_{0} & r_{5} / r_{0} \\ 1.323 & 1 . & 1 . & 1 . \\ 1.301 & .960 & .950 & 1.006 \\ 1.250 & .923 & .900 & 1.019 \\ 1.195 & .863 & .800 & 1.080 \\ 1.134 & .852 & .750 & 1.134\end{array}$

Case II. $m_{4}$ lies on the line between $m_{1}$ and $m_{3}$. In addition to the dynamical equations, the geometrical equations are

$$
r_{5}=r_{3}+r_{1}, r_{5} r_{6}^{2}=r_{4} r_{2}^{2}+r_{3} r_{1}^{2}-r_{3} r_{4} r_{5}
$$


Two points on the curve, the two end points, are easily obtained, viz.:

(a) $\quad r_{2}=r_{3}=r_{6}=r_{0}, \quad r_{5}=r_{4}+r_{0}, \quad r_{0}^{2}+r_{0} r_{4}+r_{4}^{2}=r_{1}^{2}$;

(b) $\quad r_{3}=r_{4}=r_{6}=r_{0}, \quad r_{5}=2 r_{0}, \quad r_{2}^{2}=4 r_{0}^{2}-r_{1}^{2}$.

Since $r_{2} \geqq r_{0}$, (30), it follows that the point $b$ is not real unless $r_{1} \leqq 3^{1 / 2} r_{0}$. That is, Case II offers no restriction on the areas $T_{1}$ and $S_{1}$, if $r_{1}>3^{1 / 2} r_{0}$.

The following table gives the values of the ratios at three points on this locus for $r_{1}=1.5 r_{0}$.

$\begin{array}{rrrr}r_{2} / r_{0} & r_{3} / r_{0} & r_{4} / r_{0} & r_{6} / r_{0} \\ 1.000 & 1.000 & .725 & 1.000 \\ 1.135 & .950 & .900 & .958 \\ 1.323 & 1.000 & 1.000 & 1.000\end{array}$

Case III. $m_{4}$ lies on the line between $m_{2}$ and $m_{3}$. This case is symmetrical with Case II and gives the loci which pass through and restrict the regions $S_{2}$ and $T_{2}$.

Fig. 7 shows the reduced areas $S_{1}, S_{2}, T_{1}$, and $T_{2}$ for the case $r_{1}=1.5 r_{0}$.

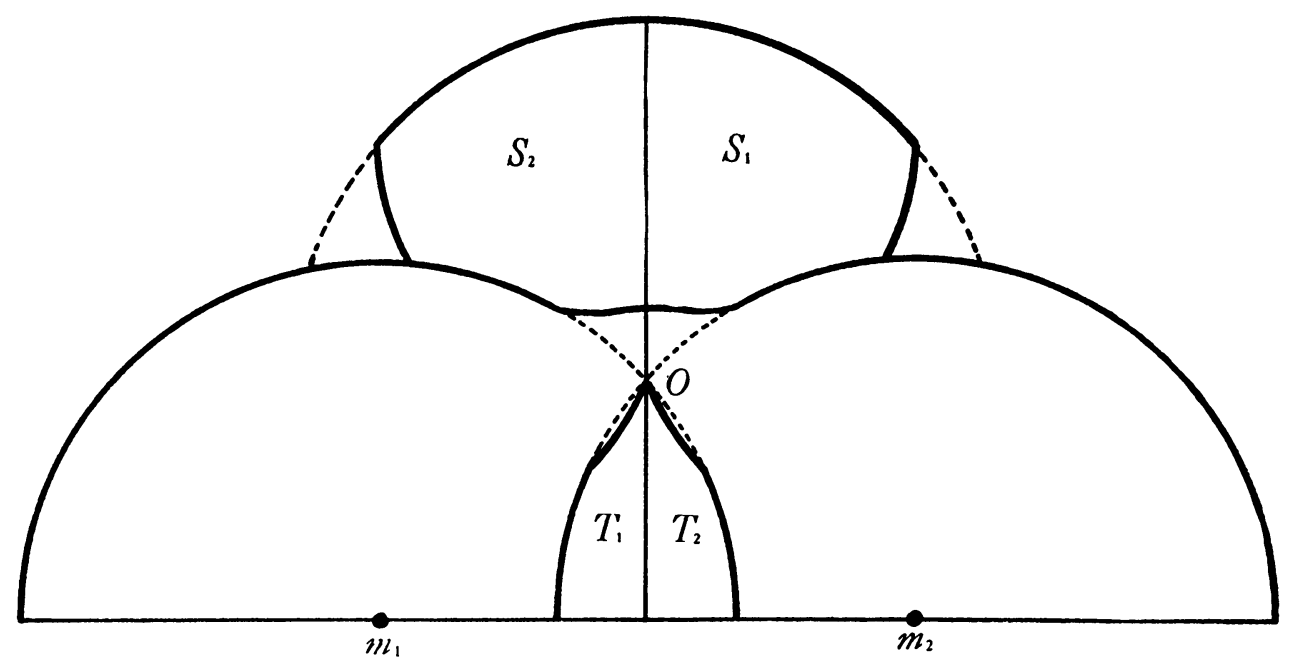

Fig. 7

18. Isosceles trapezoids. In case the convex quadrilateral is a trapezoid with $r_{3}$ parallel to $r_{1}$,

$$
\alpha_{1}=\beta_{1}=\alpha_{3}=\beta_{3}=\infty ;
$$

and

$$
\alpha_{2}=-\beta_{4}, \quad \alpha_{4}=-\beta_{2} .
$$

If the trapezoid is isosceles, one has also 


$$
r_{2}=r_{1}, \quad r_{5}=r_{6}
$$

and

$$
\alpha_{2}=\frac{r_{1}}{r_{1}-r_{3}}, \quad \beta_{2}=\frac{r_{3}}{r_{1}-r_{3}} .
$$

The second equation of (8), which is geometrical, reduces to

$$
r_{5}^{2}=r_{1} r_{3}+r_{2}^{2} .
$$

The dynamical equation (31) becomes

$$
\left(R_{1}-R_{0}\right)\left(R_{3}-R_{0}\right)=\left(R_{2}-R_{0}\right)^{2}=\left(R_{0}-R_{6}\right)^{2},
$$

from the last equality of which it follows, on extracting the square root, that, since $r_{2}<r_{0}<r_{5}$ by $\$ 14$,

$$
\begin{aligned}
& R_{2}-R_{0}=R_{0}-R_{5}>0, \text { or } \\
& R_{2}+R_{5}=2 R_{0} .
\end{aligned}
$$

A parametric solution of equations (32) and (33) can be obtained'as follows. Define the parameter $\kappa$ by the relation

$$
r_{2}=\kappa\left(r_{1} r_{3}\right)^{1 / 2}
$$

and it follows from (32) that

$$
r_{5}=\left(1+\kappa^{2}\right)^{1 / 2}\left(r_{1} r_{3}\right)^{1 / 2}
$$

also (34) becomes

$$
\frac{R_{0}}{\left(R_{1} R_{3}\right)^{1 / 2}}=\frac{1}{2}\left\{\kappa^{-3}+\left(1+\kappa^{2}\right)^{-3 / 2}\right\} .
$$

From the first equality of (33) is obtained

$$
R_{1} R_{3}-\left(R_{1}+R_{3}\right) R_{0}=R_{2}^{2}-2 R_{2} R_{0},
$$

which becomes, on using (35),

$$
\left(1-\kappa^{-6}\right) R_{1} R_{3}+2 \kappa^{-3} R_{0}\left(R_{1} R_{3}\right)^{1 / 2}-\left(R_{1}+R_{3}\right) R_{0}=0,
$$

or

$$
\left(1-\kappa^{-6}\right)+2 \kappa^{-3} \frac{R_{0}}{\left(R_{1} R_{3}\right)^{1 / 2}}-\left(\frac{R_{0}}{R_{1}}+\frac{R_{0}}{R_{3}}\right)=0 .
$$

Then, by means of (37),

$$
\left(1-\kappa^{-6}\right)+\kappa^{-3}\left\{\kappa^{-3}+\left(1+\kappa^{2}\right)^{-3 / 2}\right\}-\left(\frac{R_{0}}{R_{1}}+\frac{R_{0}}{R_{3}}\right)=0 .
$$


From (37) and (38), it is found that

where

$$
\begin{aligned}
& \frac{R_{0}}{R_{1}}+\frac{R_{0}}{R_{3}}=1+\kappa^{-3}\left(1+\kappa^{2}\right)^{-3 / 2}=1+a b, \\
& \frac{2 R_{0}}{\left(R_{1} R_{3}\right)^{1 / 2}}=\kappa^{-3}+\left(1+\kappa^{2}\right)^{-3 / 2}=a+b,
\end{aligned}
$$

$$
a=\kappa^{-3}, \quad b=\left(1+\kappa^{2}\right)^{-3 / 2} .
$$

The solution of these equations is

$$
\begin{aligned}
& r_{1}^{3}=\frac{1}{2}\left[1+a b \pm\left(\left(1-a^{2}\right)\left(1-b^{2}\right)\right)^{1 / 2}\right] r_{0}{ }^{3}, \\
& r_{3}^{3}=\frac{1}{2}\left[1+a b \mp\left(\left(1-a^{2}\right)\left(1-b^{2}\right)\right)^{1 / 2}\right] r_{0}{ }^{3},
\end{aligned}
$$

and adding

$$
r_{2}=\kappa\left(r_{1} r_{3}\right)^{1 / 2}=\left(\frac{a+b}{2 a}\right)^{1 / 3} r_{0}
$$

$$
\begin{aligned}
& r_{5}=\left(1+\kappa^{2}\right)^{1 / 2}\left(r_{1} r_{3}\right)^{1 / 2}=\left(\frac{a+b}{2 b}\right)^{1 / 3} r_{0} \\
& a=\kappa^{-3}, \quad b=\left(1+\kappa^{2}\right)^{-3 / 2}
\end{aligned}
$$

the parametric representation is complete.

A table of values of the ratios of $r_{0}, r_{2}$, and $r_{5}$ to $r_{1}$ is given in the table below.

TABLE II

\begin{tabular}{c|c|c|r|r|r}
\hline$a$ & $b$ & $r_{0} / r_{1}$ & \multicolumn{1}{c}{$r_{2} / r_{1}$} & \multicolumn{1}{c}{$r_{3} / r_{1}$} & $r_{5} / r_{1}$ \\
\hline 0 & .0000 & 1.0000 & 1.0000 & .0000 & 1.0000 \\
.1 & .0746 & 1.0001 & .9556 & .1968 & 1.0537 \\
.2 & .1286 & 1.0004 & .9370 & .3002 & 1.0855 \\
.3 & .1722 & 1.0015 & .9246 & .3831 & 1.1127 \\
.4 & .2087 & 1.0034 & .9160 & .4555 & 1.1378 \\
.5 & .2403 & 1.0066 & .9106 & .5224 & 1.1626 \\
.6 & .2680 & 1.0117 & .9082 & .5867 & 1.1880 \\
.7 & .2927 & 1.0194 & .9091 & .6515 & 1.2157 \\
.8 & .3149 & 1.0321 & .9146 & .7212 & 1.2483 \\
.9 & .3351 & 1.0531 & .9289 & .8043 & 1.2912 \\
1.0 & .3536 & 1.1390 & 1.0000 & 1.0000 & 1.4142 \\
\hline
\end{tabular}


A diagram, given in Fig. 8 , shows that as $\kappa$ increases from 1 to $\infty$ the trapezoid changes continuously from a square to an equilateral triangle. The two following theorems are evident:

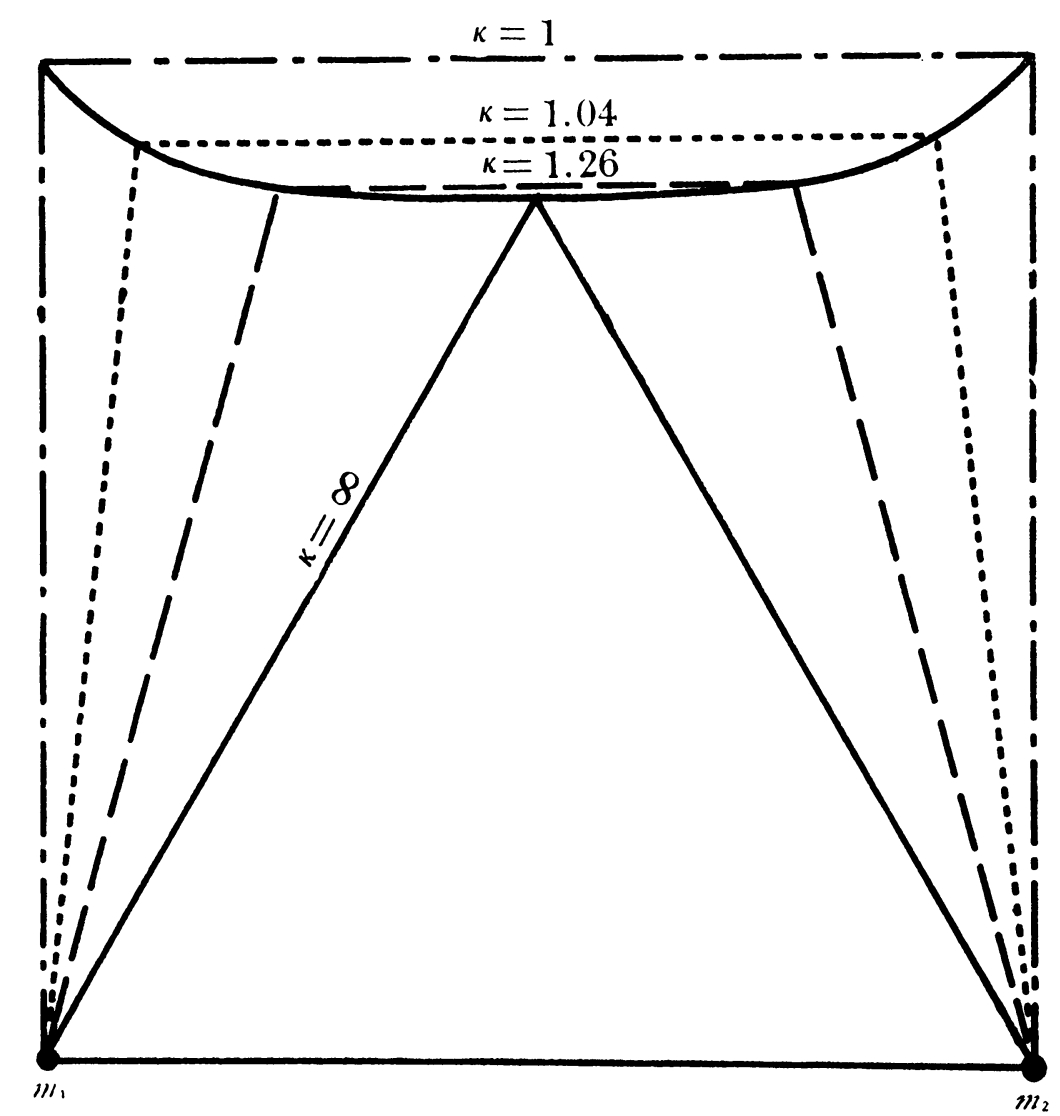

Fig. 8

Theorem. There exists one and only one isosceles trapezoid for given values of $r_{0}$ and $r_{1}$.

THEOREM. There exists one and only one isoceles trapezoid for a given value. of an interior angle, provided this angle lies between $60^{\circ}$ and $120^{\circ}$.

The mass ratios. From equations (17) it is found that

$$
\frac{m_{2}}{m_{1}}=-\frac{\alpha_{1}}{\beta_{1}} \frac{S_{4}}{S_{6}}, \quad \frac{m_{4}}{m_{3}}=-\frac{\alpha_{3}}{\beta_{3}} \frac{S_{2}}{S_{6}} .
$$

For the trapezoid, however, 


$$
\frac{\alpha_{1}}{\beta_{1}}=\frac{\alpha_{3}}{\beta_{3}}=+1
$$

and, since $R_{2}=R_{4}$ and $R_{5}=R_{6}$, equations (19) show that

$$
S_{2}=S_{4}=-S_{6}
$$

hence

$$
m_{1}=m_{2}, \text { and } m_{3}=m_{4} .
$$

Also, from (17) and (7.5),

$$
\frac{m_{3}}{m_{1}}=-\frac{\beta_{3}}{\beta_{1}} \frac{S_{1}}{S_{2}}=-\frac{\alpha_{2}}{\alpha_{4}} \frac{S_{1}}{S_{2}} .
$$

For the isosceles trapezoid $-\alpha_{4}=\beta_{2}$; hence

$$
\frac{m_{4}}{m_{2}}=\frac{m_{3}}{m_{1}}=\frac{\alpha_{2}}{\beta_{2}} \frac{S_{1}}{S_{2}}=\frac{r_{1}}{r_{3}} \frac{S_{1}}{S_{2}}=-\frac{r_{1}}{r_{3}} \frac{S_{1}}{S_{6}},
$$

which by virtue of (33) becomes

$$
\frac{m_{3}}{m_{1}}=\frac{r_{1}}{r_{3}}\left(\frac{S_{1}}{S_{3}}\right)^{1 / 2}=\left(\frac{r_{3}}{r_{1}} \cdot \frac{r_{0}^{3}-r_{1}^{3}}{r_{0}^{8}-r_{3}^{3}}\right)^{1 / 2} \text {. }
$$

For $r_{3}=0$ this ratio vanishes. As $r_{3}$ increases, both fractions of the radicand increase and have the limit unity. Hence as the trapezoid changes from the equilateral triangle to the square, the ratio $m_{3} / m_{1}$ increases steadily from zero to one. Hence

THEOREM. For every $m_{1}=m_{2}>0$ and $m_{3}=m_{4}>0$, there exists one and only one isosceles trapezoid configuration.

19. Quadrilaterals in the neighborhood of isosceles trapezoids. On returning to Fig. 4 with the assumption that $r_{3} \leqq r_{0}$, it is seen that in any given diagram, that is, $r_{1} \leqq r_{0}$ given, there exists one and only one isosceles trapezoid point in each of the regions $O a_{1} b_{1}$ and $O a_{2} b_{2}$, and each of these points is the reflection of the other in a plane $P$ which passes through $O$ and is perpendicular to $r_{1}$. For other admissible quadrilaterals $m_{4}$ lies in or on the boundary of $O a_{1} b_{1}$ and $m_{3}$ within or on the boundary of $O a_{2} b_{2}$.

Suppose $m_{4}$ approaches the boundary $O a_{1}$ and that $r_{1}<r_{0}$; then $R_{6}$ approaches $R_{0}$, and on account of the dynamical equations

$$
\left(R_{1}-R_{0}\right)\left(R_{3}-R_{0}\right)=\left(R_{2}-R_{0}\right)\left(R_{4}-R_{0}\right)=\left(R_{5}-R_{0}\right)\left(R_{6}-R_{0}\right)
$$

$R_{3}$ also tends toward $R_{0}$ and so also does $R_{4}$, provided $m_{4}$ is not approaching either of the points $O$ and $a_{1}$. That is, if $m_{1}$ approaches the arc $O a_{1}$ (end points 
excepted), the point $m_{3}$ approaches the arc $O b_{2}$, and if $m_{4}$ is on $O a_{1}$, the point $m_{3}$ is on the arc $O b_{2}$, and $r_{2}=r_{3}=r_{5}=r_{0}$. The points $m_{2}, m_{3}$, and $m_{4}$ form an equilateral triangle and the mass $m_{1}$ vanishes. Now let $m_{4}$ move along the $\operatorname{arc} a_{1} O$ toward the point $O$. The point $m_{3}$ simultaneously moves along the arc $O b_{2}$ toward the point $b_{2}$. If the point $m_{4}$ is at the point $O$, the point $m_{3}$ may be anywhere on the arc $b_{2} a_{2}$, which, extended, passes through $m_{1}$. Thus $m_{1}, m_{2}$, and $m_{3}$ lie on the arc of a circle whose center is at $m_{4}$. The corresponding three masses are all zero unless $m_{3}$ is at the end points $a_{2}$ or $b_{2}$.

Now let the point $m_{4}$ move along the arc $O b_{1}$. The point $m_{3}$ simultaneously moves along the arc $a_{2} O$. The triangle $m_{1} m_{4} m_{3}$ is equilateral, and the mass $m_{2}$ is zero, and so on. It is seen that as $m_{4}$ moves around the boundary of its region counterclockwise, $m_{3}$ also moves around the boundary of its region, but in a clockwise direction; and throughout all of the motion $r_{3}$ is constant and equal to $r_{0}$.

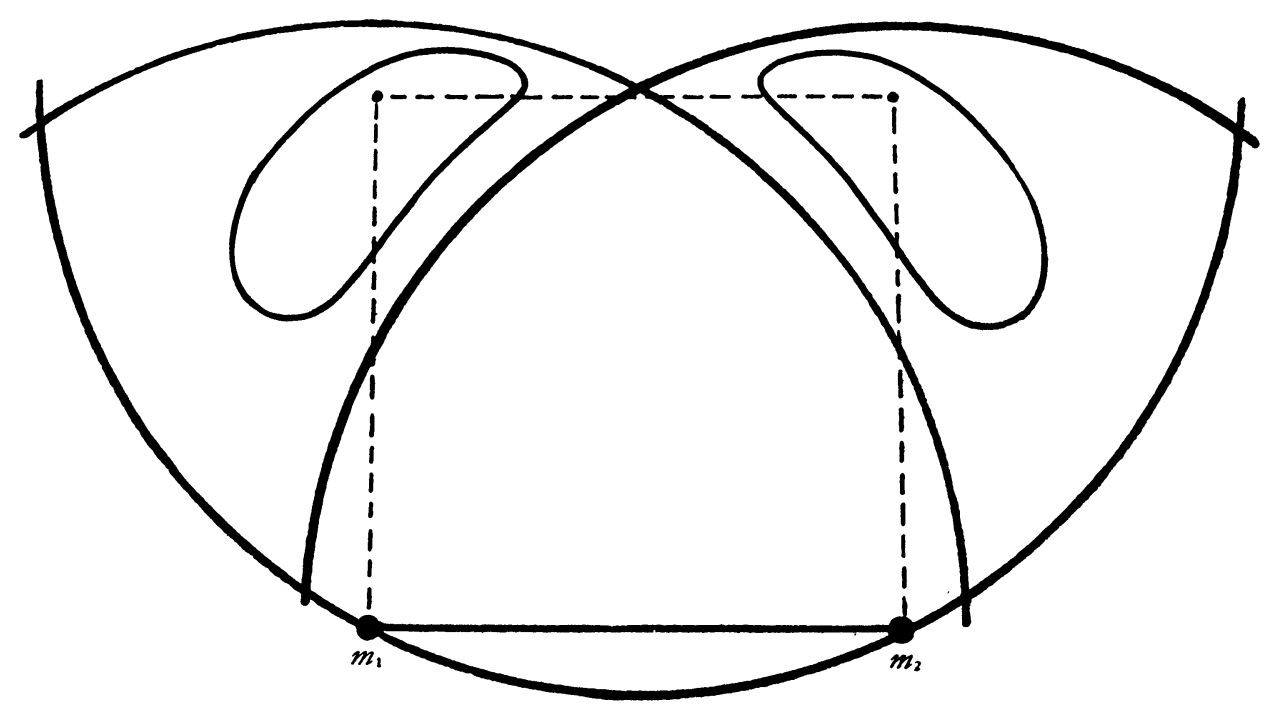

Fig. 9

If $m_{4}$ and $m_{3}$ are permitted to move in their respective regions but subject to the condition that $r_{3}<r_{0}$ is constant, it is found that each point traces a closed curve in its own region and that each of these curves is the reflection of the other in the plane $P$, Fig. 9. Thus there is a family of curves $r_{3}=C<r_{0}$. These curves shrink in length as the constant $C$ diminishes; in fact, they shrink down upon the isosceles trapezoid points as a limit (for the proof see \$21). Consequently, the smallest possible value of $r_{3}$ for given values of $r_{0}$ and $r_{1}$ is that one which belongs to the isosceles trapezoid. 
20. Conjugate curves. If the point $m_{3}$ of an admissible quadrilateral describes a curve in the region $S_{3}, m_{1}$ and $m_{2}$ remaining fixed, the point $m_{4}$ of the admissible quadrilateral also describes a curve in the region $S_{4}$. Suppose these curves are reflected in a plane which passes through $O$, Fig. 4 , and is perpendicular to $r_{1}$; then if $m_{3}$ moves along the reflected curve in $S_{3}, m_{4}$ will move along the reflected curve in $S_{4}$. These curves are conjugate curves. Do there exist conjugate curves that are unaltered by reflection? The answer is in the affirmative since the curves $r_{3}=$ const. obviously are of this type. Such a pair of curves are self conjugate. Let $C_{3}$ and $C_{4}$ be the members of a self conjugate pair; that is, $C_{3}$ is the reflection of $C_{4}$, and conversely. If $m_{3}$ lies on $C_{3}$ at a certain point $p, m_{4}$ lies on $C_{4}$ at a certain point $\pi$. The reflection of $\pi$ lies on $C_{3}$ and will be denoted by $\bar{p}$; then $p$ and $\bar{p}$ are conjugate points on $C_{3}$.

Since

$$
\left(R_{2}-R_{0}\right)\left(R_{4}-R_{0}\right)=\left(R_{0}-R_{5}\right)\left(R_{0}-R_{6}\right)
$$

for every admissible quadrilateral, and since for conjugate points $R_{\mathbf{4}}=\bar{R}_{\mathbf{2}}$ and $R_{6}=\bar{R}_{5}$, it follows that on every self conjugate curve

Since

$$
\left(R_{2}-R_{0}\right)\left(\bar{R}_{2}-R_{0}\right)=\left(R_{0}-R_{5}\right)\left(R_{0}-\bar{R}_{5}\right) .
$$

$$
\bar{r}_{5}=r_{6}, \quad \bar{r}_{2}=r_{4}, \quad \text { and } \quad \bar{r}_{4}=r_{2},
$$

for the reflected quadrilaterals, equations (8) and (9) give

$$
\begin{aligned}
& \alpha_{1} \bar{r}_{5}^{2}=-\alpha_{1} \beta_{1} r_{1}^{2}+\beta_{3}^{2} r_{3}^{2}+\beta_{1} \bar{r}_{2}^{2}, \\
& \beta_{1} r_{5}^{2}=+\alpha_{1} \beta_{1} r_{1}^{2}-\alpha_{3}^{2} r_{3}^{2}+\alpha_{1} r_{2}^{2},
\end{aligned}
$$

and the sum of these gives the relation

$$
\alpha_{1} \bar{r}_{5}^{2}+\beta_{1} r_{5}^{2}=-\left(\alpha_{3}+\beta_{3}\right) r_{2}^{2}+\left(\beta_{1} \bar{r}_{2}^{2}+\alpha_{1} r_{2}^{2}\right),
$$

which holds at the conjugate points $p$ and $\bar{p}$. If $p$ and $\bar{p}$ tend toward coincidence, $r_{3}$ tends toward parallelism with $r_{1}$, and in the limit $\alpha_{1}, \beta_{1}, \alpha_{3}$ and $\beta_{3}$ are infinite; but

$$
\lim \left(\alpha_{3} / \alpha_{1}\right)=\beta_{3} / \alpha_{1}=r_{1} / r_{3}, \quad \lim \left(\beta_{1} / \alpha_{1}\right)=+1,
$$

so that, when the two points coincide, equation (42) becomes

and equation (41) becomes

$$
r_{5}^{2}=r_{1} r_{3}+r_{2}^{2}
$$

$$
\left(R_{2}-R_{0}\right)^{2}=\left(R_{0}-R_{5}\right)^{2}
$$

Since these are the equations which define the isosceles trapezoid points, it follows that every self conjugate curve, on which the conjugate points have a point of coincidence, passes through an isosceles trapezoid point. 
As an example of a self conjugate curve of this last type consider the locus of positions of $m_{3}$ for which

$$
R_{2}-R_{0}=R_{0}-R_{5} .
$$

From the dynamical equation (41.5) it follows that the conjugate curve is defined by the relation

$$
\left(R_{4}-R_{0}\right)=\left(R_{0}-R_{6}\right),
$$

which is the reflection of the locus for $m_{3}$. Hence these curves are self conjugate and they all pass through the isosceles trapezoid points. The curves for which $r_{3}=$ const. are self conjugate, but they do not pass through the isosceles trapezoid points. They are closed curves which contain the isosceles trapezoid points in their interiors.

21. Power series solutions in the neighborhood of isosceles trapezoids. In order to investigate the properties of the self conjugate curves $r_{3}=$ const. near an isosceles trapezoid point, a power series expansion of the solutions of the geometrical and dynamical equations will be useful.

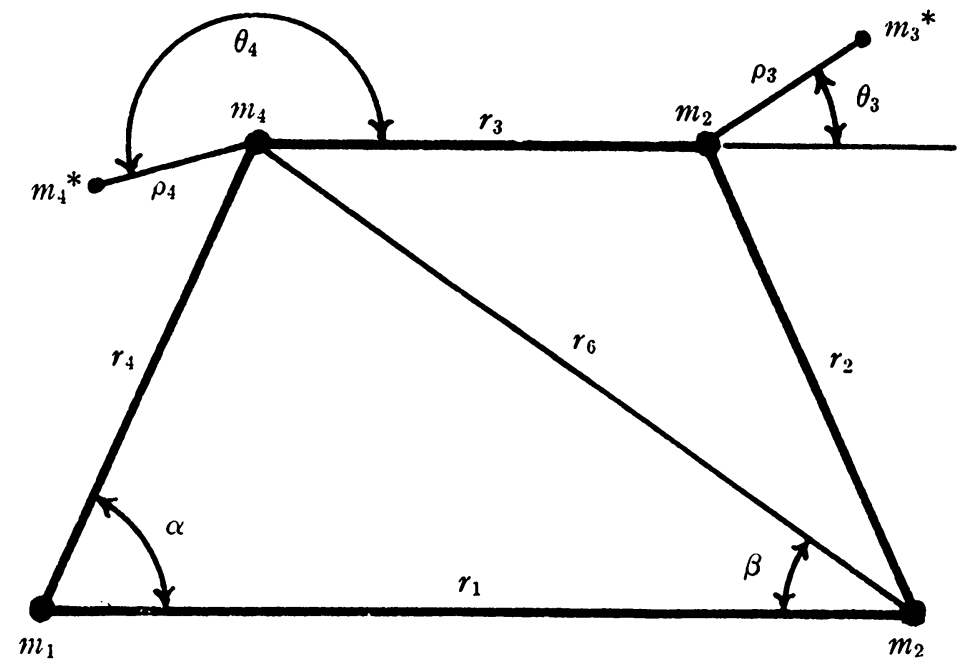

Fig. 10

Consider two points $m_{3}{ }^{*}$ and $m_{4}{ }^{*}$ of a solution which is near the isosceles trapezoid points $m_{3}$ and $m_{4}$ which are associated with the trapezoid $r_{1}, r_{2}=r_{4}$, $r_{3}, r_{5}=r_{6}$. Let the point $m_{3}^{*}$ be defined by the polar coördinates $\rho_{3}, \theta_{3}$ with origin at $m_{3}$, the polar axis being the line $m_{4}$ to $m_{3}$. Likewise let the point $m_{4}{ }^{*}$ be defined by the polar coördinates $\rho_{4}, \theta_{4}$ with the same polar axis but with origin at $m_{4}$, Fig. 10. Let $r_{1}, r_{2}{ }^{*}, r_{3}{ }^{*}, r_{4}{ }^{*}, r_{5}{ }^{*}$, and $r_{6}{ }^{*}$ be the sides and diagonals 
of the quadrilateral associated with corners $m_{1}, m_{2}, m_{3}{ }^{*}$, and $m_{4}{ }^{*}$. Let $\alpha$ be the angle between $r_{1}$ and $r_{4}$, and $\beta$ the angle between $r_{1}$ and $r_{6}$. Then

$$
\begin{aligned}
& r_{3}^{* 2}=\left(r_{3}+\rho_{3} \cos \theta_{3}-\rho_{4} \cos \theta_{4}\right)^{2}+\left(\rho_{3} \sin \theta_{3}-\rho_{4} \sin \theta_{4}\right)^{2}, \\
& r_{2}^{* 2}=r_{2}^{2}+\rho_{3}^{2}-2 r_{2} \rho_{3} \cos \left(\theta_{3}+\alpha\right), \\
& r_{4}^{* 2}=r_{2}^{2}+\rho_{4}^{2}+2 r_{2} \rho_{4} \cos \left(\theta_{4}-\alpha\right), \\
& r_{5}^{* 2}=r_{5}^{2}+\rho_{3}^{2}+2 r_{5} \rho_{3} \cos \left(\theta_{3}-\beta\right), \\
& r_{6}^{* 2}=r_{5}^{2}+\rho_{4}^{2}-2 r_{5} \rho_{4} \cos \left(\theta_{4}+\beta\right) .
\end{aligned}
$$

Since the point $m_{4}$ is uniquely defined if $m_{3}$ is given, it follows that $\rho_{4}$ vanishes if $\rho_{3}$ vanishes. If these values are substituted in the dynamical equations (41), bearing in mind, of course, that

$$
R_{i}^{*}=\left(r_{i}^{*}\right)^{-3},
$$

and the equations are then expanded in powers of $\rho_{3}$ and $\rho_{4}$ there result the two equations

$$
\begin{aligned}
& \frac{-3\left(R_{1}-R_{0}\right) R_{3}}{r_{3}}\left(\rho_{3} \cos \theta_{3}-\rho_{4} \cos \theta_{4}\right)+\cdots \\
& =\frac{3\left(R_{2}-R_{0}\right) R_{2}}{r_{2}}\left(\rho_{3} \cos \left(\theta_{3}+\alpha\right)-\rho_{4} \cos \left(\theta_{4}-\alpha\right)\right)+\cdots \\
& =\frac{3\left(R_{0}-R_{5}\right) R_{5}}{r_{5}}\left(\rho_{3} \cos \left(\theta_{3}-\beta\right)-\rho_{4} \cos \left(\theta_{4}+\beta\right)\right)+\cdots
\end{aligned}
$$

the terms independent of $\rho_{3}$ and $\rho_{4}$ vanishing by themselves, since they belong to the isosceles trapezoid solution. The solution of the linear terms of these equations gives as a first approximation to the complete solution

$$
\rho_{4}=\rho_{3}, \quad \theta_{4}=-\theta_{3} .
$$

For further development let two new variables $\kappa$ and $\lambda$ be defined as follows:

$$
\begin{aligned}
\kappa & =\frac{\rho_{4}-\rho_{3}}{\rho_{3}}=\kappa_{1} \rho+\kappa_{2} \rho^{2}+\cdots, \\
\lambda & =\theta_{3}+\theta_{4}=\lambda_{1} \rho+\lambda_{2} \rho^{2}+\cdots, \\
\rho_{3} & =\rho, \quad \theta_{3}=\theta,
\end{aligned}
$$

since $\kappa$ and $\lambda$ are evidently expansible as a convergent power series in $\rho$, the coefficients being functions of $\theta$. With these variables, $\rho, \theta, \kappa, \lambda$, the expansion of the first equation of (43), up to and including terms of the second degree in $\rho$, is 


$$
r_{3}^{* 2}=r_{3}^{2}+2 r_{3}(-\rho \kappa \cos \theta+\rho \lambda \sin \theta)+4 \rho^{2} \sin ^{2} \theta+\cdots ;
$$

and similar expansions can be obtained for the remaining sides and diagonals. When these expansions are substituted into the dynamical equations (41), not only the terms independent of $\rho$ are satisfied by themselves, but the linear terms also. Since $\kappa$ and $\lambda$ occur only in terms of at least the second degree in $\rho, \kappa$, and $\lambda$, each of which contains $\rho$ as a factor, the factor $\rho$ can be divided out, and there remain the two equations

$$
\begin{aligned}
& \frac{\left(R_{1}-R_{0}\right) R_{3}}{r_{8}}\left[\kappa \cos \theta-\lambda \sin \theta-\frac{2 \rho \sin ^{2} \theta}{r_{3}}+\cdots\right] \\
& =\frac{\left(R_{2}-R_{0}\right) R_{2}}{r_{2}}\left[-\kappa \cos (\theta+\alpha)+\lambda \sin (\theta+\alpha)-\frac{\rho\left(1-5 \cos ^{2}(\theta+\alpha)\right)}{r_{2}}\right] \\
& \quad-3 \frac{R_{2}{ }^{2}}{r_{2}{ }^{2}} \rho \cos ^{2}(\theta+\alpha)+\cdots \\
& =\frac{\left(R_{0}-R_{5}\right) R_{5}}{r_{5}}\left[-\kappa \cos (\theta-\beta)-\lambda \sin (\theta-\beta)+\frac{\rho\left(1-5 \cos ^{2}(\theta-\beta)\right)}{r_{5}}\right] \\
& \quad-3 \frac{R_{5}{ }^{2}}{r_{5}{ }^{2}} \rho \cos ^{2}(\theta-\beta)+\cdots .
\end{aligned}
$$

These two equations can be solved for $\kappa$ and $\lambda$ uniquely as a power series in $\rho$, as indicated in (45), provided the determinant of the coefficients of $\kappa$ and $\lambda$ is different from zero. This determinant is

$$
\begin{aligned}
& \frac{\left(R_{2}-R_{0}\right)\left(R_{0}-R_{5}\right) R_{2} R_{5}}{r_{2} r_{5}} \sin (\alpha+\beta)+\frac{\left(R_{1}-R_{0}\right)\left(R_{0}-R_{5}\right) R_{3} R_{5}}{r_{3} r_{5}} \sin \beta \\
& +\frac{\left(R_{1}-R_{0}\right)\left(R_{2}-R_{0}\right) R_{3} R_{2}}{r_{3} r_{2}} \sin \alpha .
\end{aligned}
$$

In $\$ 16$ it was shown that $60^{\circ}<\alpha<120^{\circ}$ and $\beta<60^{\circ}$. Consequently $\alpha+\beta$ $<180^{\circ}$, and all of the terms of the determinant are positive. It cannot, therefore, vanish. The coefficients in the expansions of equations (45) can be computed, but, for the sake of brevity, the computations will be omitted. They are found to be periodic functions of $\theta$ with the period $2 \pi$, a result that could have been anticipated from the geometrical relations between the conjugate points. If these expansions are substituted in (46), and the coefficients simplified by simple trigonometric relations between the sides and angles of the trapezoid, it is found that 
$r_{3}^{*}=r_{3}+0 \cdot \rho$

(48)

$$
\begin{aligned}
& +\frac{r_{3}\left(5 R_{0}-2 R_{2}\right) \cos ^{2}(\theta+\alpha)+r_{3}\left(5 R_{0}-2 R_{5}\right) \cos ^{2}(\theta-\beta)+2 r_{1}\left(R_{2}-R_{0}\right) \sin ^{2} \theta}{R_{3}\left(R_{1}-R_{0}\right)\left(R_{2}^{2}+R_{5}^{2}\right)+r_{1} r_{3}\left(R_{2}-R_{0}\right)} \rho^{2} \\
& +\ldots
\end{aligned}
$$

From the relations between the sides and diagonals of admissible quadrilaterals, $\$ 14$, and equation (34), it follows that all of the coefficients in equation (48) are positive. Therefore for values of $\rho$ different from zero $r_{3}{ }^{*}$ is greater than $r_{3}$. This establishes analytically the limit property of the isosceles trapezoid points discussed in $\$ 19$.

The locus of $m_{3}{ }^{*}$, for $r_{3}{ }^{*}=$ const., up to terms of the second degree in $\rho$, is

$$
\begin{aligned}
& \rho^{2}\left[r_{3}\left(5 R_{0}-2 R_{2}\right) \cos ^{2}(\theta+\alpha)+r_{3}\left(5 R_{0}-2 R_{5}\right) \cos ^{2}(\theta-\beta)+2 r_{1}\left(R_{2}-R_{0}\right) \sin ^{2} \theta\right] \\
& \quad+\cdots=\text { a constant. }
\end{aligned}
$$

The expression within the bracket is a homogeneous quadratic function of $\sin \theta$ and $\cos \theta$ that does not vanish. Therefore for $\rho$ sufficiently small the self conjugate curves $r_{3}=$ const. are approximately ellipses with centers at the isosceles trapezoid points. The conjugate ellipse described by $m_{4}{ }^{*}$ is, of course, the reflection of that described by $m_{3}{ }^{*}$, but if $m_{3}{ }^{*}$ moves in its ellipse clockwise, $m_{\mathbf{4}}{ }^{*}$ moves in its ellipse counterclockwise, so that the position of $m_{4}^{*}$ in its ellipse is not the reflection of $m_{3}{ }^{*}$ in its ellipse.

22. Masses associated with admissible quadrilaterals. It was shown in $\$ \$ 14$ and 17 that the ratios of the masses which are associated with a given admissible quadrilateral and a given $r_{0}$ are uniquely determined by equations (26). Indeed, if the six sides of the quadrilateral are given, $r_{0}$ itself is uniquely determined by equation (18), unless all three of the expressions for $R_{0}$ take an indeterminate form, and this can happen only if

or

$$
R_{1}=R_{2}=R_{5} \text { and } R_{2}=R_{4}=R_{6}
$$

$$
r_{1}=r_{2}=r_{5} \text { and } r_{2}=r_{4}=r_{6}
$$

In this case $r_{0}$ is entirely arbitrary; the masses $m_{1}, m_{2}$, and $m_{3}$ are equal and lie at the vertices of an equilateral triangle; the mass $m_{4}$ is arbitrary but it is placed at the center of the equilateral triangle which is formed by the other three masses.

THEOREM. Associated with each admissible quadrilateral there is one and only one set of mass ratios, with the single exception of three equal masses at the vertices of an equilateral triangle and a fourth arbitrary mass at the center of grainity of the other three. 
With one exception, if an admissible quadrilateral is given the mass ratios are uniquely determined. If the masses are given, does there necessarily exist an admissible quadrilateral for them, and, if so, is this quadrilateral unique?

Consider first convex quadrilaterals for which the condition

$$
r_{0}>r_{1}>0
$$

holds, and suppose $r_{0}$ is given. For every position of $m_{3}$ in the region $O a_{2} b_{2}$, Fig. 4, there exists one and only one position for $m_{4}$, and that position lies in the region $O b_{1} a_{1}$. The mass ratios $m_{1}: m_{2}: m_{3}: m_{4}$ can be regarded as functions of the position of $m_{3}$, for both the mass ratios and the ratios $\alpha_{i} / \beta_{i}, i=1,2,3,4$, are continuous single-valued functions of the position of $m_{3}$. The ratios

$$
\frac{\alpha_{i}}{\beta_{i}}=0, \text { or } \infty \quad(i=1,2,3,4)
$$

implies that at least three of the bodies are in a straight line. Hence it is evident from Fig. 4 that for every $r_{1} \neq 0$ that is less than $r_{0}$ the ratios $\alpha_{i} / \beta_{i}$ have a positive finite upper bound and a positive non-zero lower bound. If $m_{3}$ approaches any point on the arc $O b_{2}$ (the point $O$ itself excepted), the ratio $m_{1}: m_{2}$ approaches $0: 1$, by equation (27). If $m_{3}$ approaches any point of the $\operatorname{arc} O a_{2}$ (the point $O$ again excepted), the ratio $m_{1}: m_{2}$ approaches $0: 1$. Therefore there exists a curve $C_{3}$ which starts at $O$ and terminates on the arc $a_{2} b_{2}$ on which the ratio $m_{1}: m_{2}=k_{12}$ is an arbitrarily given positive constant.

As $m_{3}$ moves along the curve $C_{3}$, the point $m_{4}$ moves along a certain curve $C_{4}$ in the region $O b_{1} a_{1}$. If $m_{3}$ approaches $O$ on $C_{3}$ the point $m_{4}$ approaches a definite point on the arc $a_{1} b_{1}$, and the ratio $m_{3}: m_{4}$ approaches $1: 0$. If $m_{3}$ approaches the arc $a_{2} b_{2}$ along the curve $C_{3}$, the ratio $m_{3}: m_{4}$ approaches $0: 1$. Hence there exists a point on the curve $C_{3}$ at which $m_{3}: m_{4}=k_{34}$, an arbitrarily given constant. Hence

THEOREM. For every $r_{0}$ and $r_{1}$ such that $r_{0}>r_{1}>0$, there exists at least one admissible quadrilateral for which

$$
\frac{m_{1}}{m_{2}}=k_{12} \text { and } \frac{m_{3}}{m_{4}}=k_{34},
$$

where $k_{12}$ and $k_{34}$ are arbitrarily given positive constants.

From the symmetry of the figure, it follows that for every $r_{3}<r_{0}$ there exists an admissible quadrilateral for which

$$
\frac{m_{1}}{m_{2}}=k_{12} \text { and } \frac{m_{3}}{m_{4}}=k_{34} \text {. }
$$


Now consider the series of admissible convex quadrilaterals for which

$$
\frac{m_{1}}{m_{2}}=k_{12} \text { and } \frac{m_{3}}{m_{4}}=k_{34},
$$

as, $r_{0}$ remaining fixed, $r_{1}$ tends toward zero. It follows immediately from the geometry of the quadrilateral, Fig. 1, that as $r_{1}$ tends toward zero, $r_{5}$ tends toward $r_{2}$, and $r_{6}$ tends toward $r_{4}$. But in every admissible convex quadrilateral

$$
r_{2}<r_{0}<r_{5} \text { and } r_{4}<r_{0}<r_{6}
$$

Consequently, at the limit

$$
r_{2}=r_{4}=r_{5}=r_{6}=r_{0}
$$

and since

$$
\left(R_{1}-R_{0}\right)\left(R_{3}-R_{0}\right)=\left(R_{2}-R_{0}\right)\left(R_{4}-R_{0}\right),
$$

the left member tends toward zero just as the right member does. But $R_{1}$ tends toward infinity; hence $R_{3}$ tends toward $R_{0}$, which is the same as saying that $r_{3}$ tends toward $r_{0}$. Consequently as $r_{1}$ tends toward zero, all of the remaining sides and the diagonals tend toward the value $r_{0}$.

The functions $\alpha_{2}$ and $\beta_{4}$, however, tend toward zero as $r_{1}$ diminishes; for the point $A$ in Fig. 1 tends toward coincidence with the point $m_{2}$. But since, $\$ 5$,

it is seen that

$$
r_{1} a_{1}+\alpha_{2} r_{2} a_{2}=\beta_{4} r_{4} a_{4}
$$

$$
a_{1}+\frac{\alpha_{2}}{r_{1}} r_{2} a_{2}=\frac{\beta_{2}}{r_{1}} r_{4} a_{4},
$$

and consequently as $r_{1}$ tends toward zero, the ratios $\alpha_{2} / r_{1}$ and $\beta_{4} / r_{1}$ tend toward limits that are not zero. For the angles between the unit vectors $\boldsymbol{a}_{1}$ and $a_{2}$, and between $\boldsymbol{a}_{4}$ and $\boldsymbol{a}_{1}$ for admissible convex quadrilaterals, always lie between $60^{\circ}$ and $120^{\circ}$, and the angle between $a_{4}$ and $a_{2}$ tends toward the definite limit $120^{\circ}$. The common limit of $r_{2}$ and $r_{4}$ is $r_{0}$, and the coefficient of $a_{1}$ in the above equation is unity. Hence zero is not a limit for either $\alpha_{2} / r_{1}$ or $\beta_{4} / r_{1}$.

Now, by equations (26),

$$
\frac{m_{1}}{m_{3}}=\frac{\alpha_{4} \beta_{2}}{\beta_{4} \alpha_{2}} \frac{R_{3}-R_{0}}{R_{1}-R_{0}} \frac{m_{4}}{m_{2}}=\frac{\alpha_{4} \beta_{2}}{\left(\frac{\beta_{4} \alpha_{2}}{r_{1}^{2}}\right)} \frac{r_{1}\left(R_{3}-R_{0}\right)}{1-r_{1}^{3} R_{0}} \frac{m_{4}}{m_{2}} .
$$


Since the limit of $\alpha_{2}$ and $\beta_{4}$ is zero, the limits of $\beta_{2}$ and $\alpha_{4}$ are -1 and +1 respectively. Hence, as $r_{1}$ tends toward zero, $r_{0}$ remaining fixed, the limit of

$$
\frac{m_{1} m_{2}}{m_{3} m_{4}}=0 .
$$

In a similar manner it is shown that if $r_{1}$ tends toward $r_{0}$, then $r_{3}$ tends toward zero, and the limit of

$$
\frac{m_{3} m_{4}}{m_{1} m_{2}}=0 .
$$

It follows, therefore, that for some value of $r_{1}$ in the interval

$$
0 \leqq r_{1} \leqq r_{0}
$$

there exists an admissible convex quadrilateral for which

$$
\frac{m_{1}}{m_{2}}=k_{12}, \quad \frac{m_{3}}{m_{4}}=k_{34}, \text { and } \frac{m_{1} m_{2}}{m_{3} m_{4}}=k,
$$

where $k_{12}, k_{34}$, and $k$ are arbitrarily specified constants. It will be observed that it is not proved that there exists but one such value of $r_{1}$.

This result can be expressed as follows:

THEOREM. For every four given masses and assigned order there exists at least one admissible convex quadrilateral.

A corresponding theorem for concave quadrilaterals has not been proved.

University of Chicago,

Chicago, Ill. 